\title{
The Impact of Diagnostic Criteria for Gestational Diabetes Mellitus on Adverse Maternal Outcomes: A Systematic Review and Meta-Analysis
}

\author{
Fahimeh Ramezani Tehrani ${ }^{1}$, Marzieh Saei Ghare Naz ${ }^{1}$, Razieh Bidhendi Yarandi $^{1}$ and \\ Samira Behboudi-Gandevani ${ }^{2, *(D)}$ \\ 1 Reproductive Endocrinology Research Center, Research Institute for Endocrine Sciences, Shahid Beheshti \\ University of Medical Sciences, Tehran 1985717413, Iran; ramezani@endocrine.ac.ir (F.R.T.); \\ saeigarenaz@gmail.com (M.S.G.N.); razi_bidhendi@yahoo.com (R.B.Y.) \\ 2 Faculty of Nursing and Health Sciences, Nord University, 8049 Bodø, Norway \\ * Correspondence: samira.behboudi-gandevani@nord.no; Tel.: +47-75517670
}

check for updates

Citation: Ramezani Tehrani, F.; Naz, M.S.G.; Yarandi, R.B.; Behboudi-

Gandevani, S. The Impact of

Diagnostic Criteria for Gestational

Diabetes Mellitus on Adverse

Maternal Outcomes: A Systematic

Review and Meta-Analysis. J. Clin.

Med. 2021, 10, 666. https://doi.org/

$10.3390 / \mathrm{jcm} 10040666$

Academic Editor:

Rinat Gabbay-Benziv

Received: 12 January 2021

Accepted: 3 February 2021

Published: 9 February 2021

Publisher's Note: MDPI stays neutral with regard to jurisdictional claims in published maps and institutional affiliations.

Copyright: (c) 2021 by the authors. Licensee MDPI, Basel, Switzerland. This article is an open access article distributed under the terms and conditions of the Creative Commons Attribution (CC BY) license (https:// creativecommons.org/licenses/by/ $4.0 /)$.

\begin{abstract}
This systematic review and meta-analysis aimed to examine the impact of different gestational-diabetes (GDM) diagnostic-criteria on the risk of adverse-maternal-outcomes. The search process encompassed PubMed (Medline), Scopus, and Web of Science databases to retrieve original, population-based studies with the universal GDM screening approach, published in English language and with a focus on adverse-maternal-outcomes up to January 2020. According to GDM diagnostic criteria, the studies were classified into seven groups. A total of 49 population-based studies consisting of 1,409,018 pregnant women with GDM and 7,667,546 non-GDM counterparts were selected for data analysis and knowledge synthesis. Accordingly, the risk of adverse-maternal-outcomes including primary-cesarean, induction of labor, maternal-hemorrhage, and pregnancy-related-hypertension, overall, regardless of GDM diagnostic-criteria and in all diagnostic-criteria subgroups were significantly higher than non-GDM counterparts. However, in meta-regression, the increased risk was not influenced by the GDM diagnostic-classification and the magnitude of the risks among patients, using the IADPSG criteria-classification as the most strict-criteria, was similar to other criteria. In conclusion, a reduction in the diagnostic-threshold increased the prevalence of GDM, but the risk of adverse-maternal-outcome was not different among those women who were diagnosed through more or less intensive strategies. Our review findings can empower health-care-providers to select the most cost-effective approach for the screening of GDM among pregnant women.
\end{abstract}

Keywords: adverse maternal outcomes; diagnostic criteria; gestational diabetes; meta-analysis

\section{Introduction}

Gestational diabetes mellitus (GDM) is one of the most prevalent endocrinopathies during pregnancy and affects $4-12 \%$ of all pregnancies depending on the type of diagnostic criteria as well as the prevalence of associated risk factors such as type 2 diabetes (T2DM), body mass index (BMI), advanced maternal age, and ethnicity [1-4]. Chronic disturbances in maternal $\beta$-cell, release of diabetogenic peptides from the placenta, and hormones may play a key role in the pathophysiology of GDM [5]. However, GDM is strongly associated with a higher risk of adverse pregnancy outcomes [6,7], lifelong risk of abnormal glucose tolerance, and diabetes later in life [8,9]. However, appropriate treatment strategies for GDM including lifestyle modifications and pharmacotherapy such as insulin or metformin can significantly decrease related adverse outcomes. In addition, inositol as a nutritional supplementation has been shown to improve glycemic homeostasis during pregnancy and prevent GDM $[9,10]$.

There are ongoing debates regarding the optimum GDM screening strategy. In this respect, the risk of developing postpartum T2DM among women with a history of GDN 
has been used as the first criteria for the definition of GDM; subsequently, GDM has been defined based on adverse pregnancy outcomes [11] after the Hyperglycemia and Adverse Pregnancy Outcomes' (HAPO) study, which has shown a linear continuous association between the increasing values of maternal blood glucose and adverse pregnancy outcomes [12]. The International Association of Diabetes in Pregnancy Study Group (IADPSG) [13] and the World Health Organization (WHO) [14] have recommended 75-g oral glucose tolerance test (75 g-OGTT), as the diagnostic criteria for GDM. Although this definition is one of the lowest thresholds for GDM definition, the evidence supporting this endorsement is consensus-based.

Previous reviews have shown associations between GDM and adverse perinatal outcomes just based on the WHO and IADPSG criteria [6] or the IADPSG and Carpenter and Coustan definition [15].

Lack of an evidence-based international definition of GDM may potentially influence the accurate estimation of the risk of adverse maternal outcomes. Therefore, this systematic review and meta-analysis examined the impact of various GDM criteria on the risk of adverse maternal outcomes.

\section{Materials and Methods}

The standard guideline for conducting and reporting meta-analysis [16] was used in this review. The review objectives were as follows:

- To study the pooled risk of adverse maternal outcomes among pregnant women with GDM compared to non-GDM counterparts, regardless of diagnostic criteria;

- To study the pooled risk of adverse maternal outcomes among pregnant women with GDM compared to non-GDM women, according to the various diagnostic criteria;

- To study the association between adverse maternal outcomes and GDM criteria.

\subsection{Eligibility Criteria}

Satisfaction with fulfilling the following criteria was considered for selecting studies: universal screening of GDM; having a population-based design; full description of the GDM screening method and glucose cutoff point in the screening test; reporting the prevalence or risk of short-term maternal outcomes in both GDM and non-GDM groups. Non-original studies and also those with unclear data or insufficient information about the review topic were excluded.

\subsection{Search Strategy}

The authors systematically searched on online databases such as PubMed [including Medline], Scopus, and Web of Science to retrieve original studies published in English on the prevalence, incidence, and risk of adverse maternal outcomes among women with GDM up to January 2020, using the following keywords: (adverse pregnancy outcomes OR pregnancy outcomes OR pregnancy complications OR preeclampsia OR pregnancy-induced hypertension OR gestational hypertension OR PIH OR hemorrhage OR postpartum hemorrhage OR PPH OR placenta abruption OR decolman OR placenta previa OR antepartum hemorrhage OR maternal weight gain OR pregnancy weight gain OR induction of labor OR labor induction OR induced labor OR cesarean sections OR c-section OR abdominal deliveries) AND (pregnancy-induced diabetes OR diabetes in pregnancy OR gestational diabetes mellitus OR gestational diabetes OR GDM).

In addition, the reference lists of the included articles and relevant reviews were manually searched to enhance the possibility of identifying eligible studies.

\subsection{Study Selection and Data Extraction}

Two investigators (M.S.G.N, S.B.G) independently selected manuscripts by the title, abstract, and full text. Next, the following information from each study were extracted: the first author's name, publication year, study location, sample size, research design, GDM screening characteristics including the screening strategy, details of GDM definition, quality 
assessment, and outcome measurements in terms of number and prevalence, incidence, or risk of adverse events.

\subsection{Study Subgroups and Outcomes of Study}

The studies were classified into seven sub-groups according to the GDM definition as follows:

(i) IADPSG criteria, one step screening with oral glucose tolerance test (2 h, $75 \mathrm{~g}$ GTT); GDM diagnosis: any of the given values are met or exceeded (fasting: $92 \mathrm{mg} / \mathrm{dL}$, BS-1 h: $180 \mathrm{mg} / \mathrm{dL}, \mathrm{BS}-2 \mathrm{~h}: 153 \mathrm{mg} / \mathrm{dL})$;

(ii) One step screening with $2 \mathrm{~h}, 75 \mathrm{~g}$ OGTT. GDM diagnosis: any of the given valued are met or exceeded (fasting $100 \mathrm{mg} / \mathrm{dL}, 2 \mathrm{h:} 144 \mathrm{mg} / \mathrm{dL}$ );

(iii) One step screening with $2 \mathrm{~h}, 75 \mathrm{~g}$ OGTT. GDM diagnosis: any of the given valued are met or exceeded (fasting: $110 \mathrm{mg} / \mathrm{dL}, 2 \mathrm{h:} 140 \mathrm{mg} / \mathrm{dL}$ );

(iv) Group 4, one step screening with 2 h, $75 \mathrm{~g}$ OGTT. GDM diagnosis: any of the given values are met or exceeded (fasting $100 \mathrm{mg} / \mathrm{dL}$, BS $2 \mathrm{h:} 162 \mathrm{mg} / \mathrm{dL}$ );

(v) Two step screening with 1 h-50 g Glucose challenge test ( 1 h-50 g-GCT), values > $140 \mathrm{mg} / \mathrm{dL}$ following $100 \mathrm{~g}$ OGTT. GDM diagnosis: two values are met or exceeded (fasting: 95 mg/dL, BS-1 h: 180 mg/dL, BS-2 h: 155 mg/dL, BS-3 h: 140 mg/dL or two step screening with $1 \mathrm{~h}-50 \mathrm{~g}-\mathrm{GCT}$, values > $140 \mathrm{mg} / \mathrm{dL}$ following $75 \mathrm{~g}$ OGTT. GDM diagnosis: two values are met or exceeded (fasting: $95 \mathrm{mg} / \mathrm{dL}, \mathrm{BS}-1 \mathrm{~h}: 180 \mathrm{mg} / \mathrm{dL}$, BS-2 h: $155 \mathrm{mg} / \mathrm{dL}$, BS-3 h: $140 \mathrm{mg} / \mathrm{dL}$ );

(vi) Two step screening with $1 \mathrm{~h}-50 \mathrm{~g}-\mathrm{GCT}$, values $>140 \mathrm{mg} / \mathrm{dL}$ following $100 \mathrm{~g}$ OGTT. GDM diagnosis: two values are met or exceeded (fasting: $105 \mathrm{mg} / \mathrm{dL}, \mathrm{BS}-1 \mathrm{~h}$ : $155 \mathrm{mg} / \mathrm{dL}, \mathrm{BS}-2 \mathrm{h:} 165 \mathrm{mg} / \mathrm{dL}, \mathrm{BS}-1 \mathrm{h:} 145 \mathrm{mg} / \mathrm{dL})$;

(vii) One step screening with $75 \mathrm{~g}$ OGTT. GDM diagnosis: any of the given valued are met or exceeded (fasting: $128 \mathrm{mg} / \mathrm{dL}, \mathrm{BS} 2 \mathrm{~h}: 140 \mathrm{mg} / \mathrm{dL}$ ).

The adverse maternal outcomes in this review were primary cesarean; gestational weight gain; induction of labor; maternal hemorrhage including antepartum or postpartum hemorrhage, placenta previa, placenta abruption; hypertension-related pregnancy including pregnancy-induced hypertension, preeclampsia, eclampsia.

For quality appraisal, the modified Newcastle-Ottawa Quality Assessment Scale was used [17]. As a validated and standard scale, it assessed nonrandomized studies for inclusion to meta-analyses in terms of the selection of participants, comparability of the study, and assessment of outcomes. Scores above 6, 3-5, and below 3 were interpreted as high, moderate, and low quality, respectively.

The (ROBINS) tool in non-randomized studies of interventions and observational studies was used for assessing the risk of bias [18], which has been recommended by the Cochrane [19]. Five domains of (i) assessment of exposure, (ii) development of outcome of interest in case and controls, (iii) selection of cases, (iv) selection of cases, and (v) control of prognostic variable in cross-sectional studies and 7 domains of (i) selection of exposed and nonexposed cohort, (ii) assessment of exposure, (iii) presence of outcome of interest at the start of the study, (iv) control of prognostic variables, (v) assessment of the presence or absence of prognostic factors, (vi) assessment of outcome, (vii) adequacy of follow up for cohort studies were used for appraisal. The authors classified their judgment on the quality of each study into high risk, unclear risk, or low risk of bias [19].

\subsection{Statistical Analysis}

The Stata version 12 was used for data analysis. Heterogeneity was estimated by $\mathrm{I}^{2}$ statistic. The pooled effect size including pooled odds ratio and pooled standardized mean differences of events was calculated using the fixed or random-effects models with MantelHaenszel method. Publication bias was evaluated using Begg's test. The association between the risk of adverse outcome of GDM and its diagnostic criteria as a potential source of heterogeneity was assessed using meta-regression. IADPSG definition criteria 
were used as the reference group for the comparison. All tests were two-sided and $p<0.05$ was considered statistically significant.

\section{Results}

\subsection{Literature Search Results and Quality assessment}

Figure 1 illustrates the flow diagram of the search strategy and study selection.
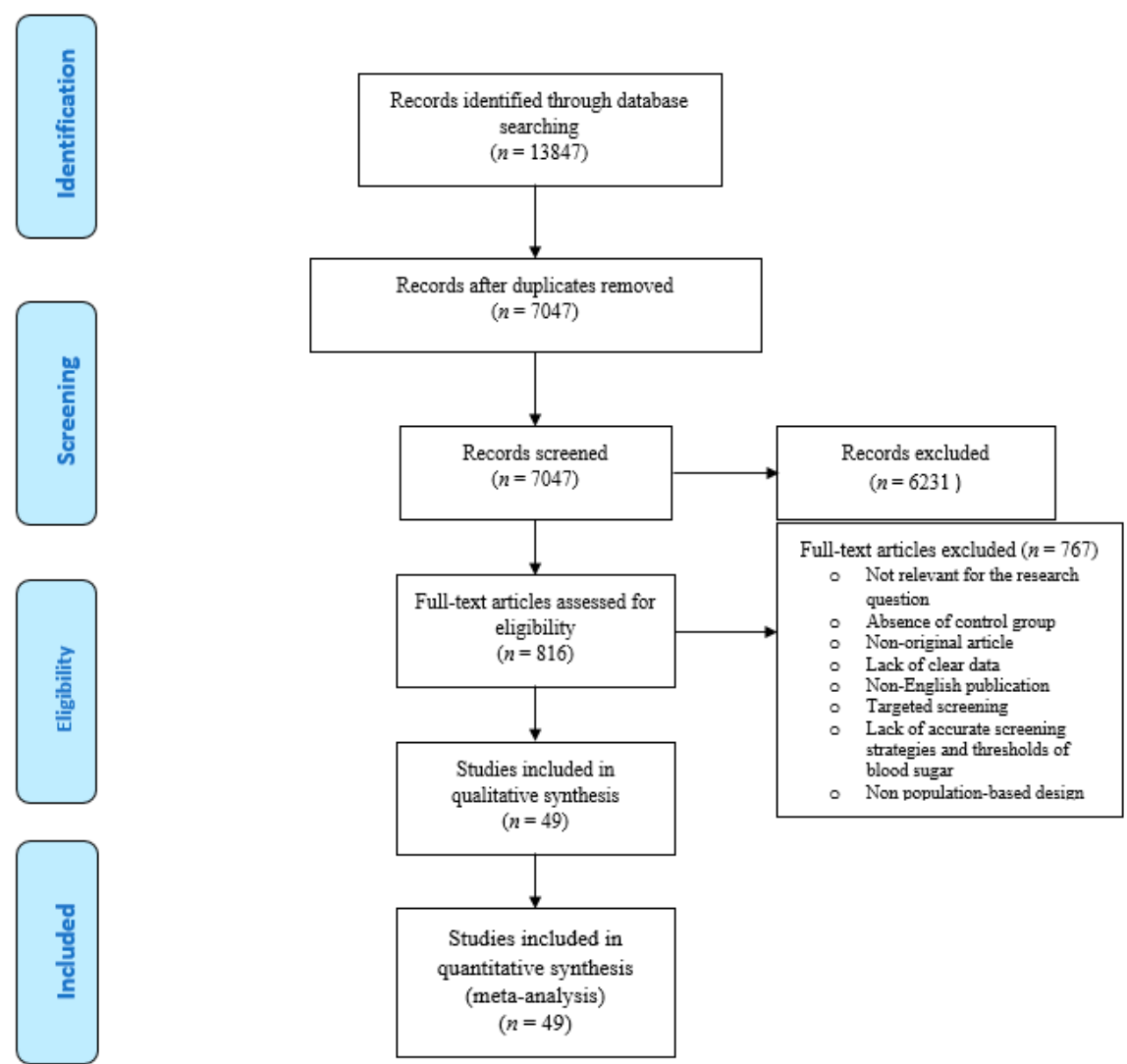

Figure 1. Flow diagram of literature search.

The search led to 13,847 studies of which 49 studies had the required inclusion criteria and were included in the meta-analysis. The studies' populations were 1,409,018 pregnant women with GDM and 7,667,546 non-GDM counterparts. Table 1 shows the summary of the studies evaluating the risk of adverse maternal outcomes among GDM and non-GDM populations. 
Table 1. Characteristics of studies assessing the adverse pregnancy outcome in gestational diabetes mellitus (GDM) and non-GDM population.

\begin{tabular}{|c|c|c|c|c|c|}
\hline Author, Year & Country & $\begin{array}{c}\text { GDM } \\
\text { Diagnostic Criteria }\end{array}$ & $\begin{array}{l}\text { GDM } \\
\text { Characteristics * }\end{array}$ & Non-GDM Characteristics * & $\begin{array}{l}\text { Adverse Maternal Outcome in Women with vs. without } \\
\text { GDM, \% or Mean (SD) }\end{array}$ \\
\hline Capula et al., 2013 & Italy & IADPSG & $\mathrm{n}=171$, Age: 30.8 (3.2), BMI: 22.8 (1.9) & n = 367, Age: 29.3 (3.5), BMI: 21.4 (2.0) & $\begin{array}{l}\text { Hypertension: } 4.1 \text { vs. } 1.6 \text {; Preeclampsia: } 2.9 \text { vs. } 1.4 \text {; Labor } \\
\text { induction: } 1.2 \text { vs. } 0.3 \text {; gestational weight gain: } 10.3 \text { (3.4) vs. } 8 \\
\text { (2.8); Primary cesarean section: } 29.8 \text { vs. } 15.3\end{array}$ \\
\hline Karmon et al., 2009 & Israel & $\mathrm{CC}$ & $\mathrm{n}=10,227$ & $\mathrm{n}=174,029$ & $\begin{array}{l}\text { Hypertensive disorders: } 11.6 \text { vs. } 5.5 \text { Abruption: } 0.8 \text { vs. } 0.7 \text {; Labor } \\
\text { induction: } 42.1 \text { vs. } 27.0 .\end{array}$ \\
\hline Moses et al., 1995 & Australia & ADIPS & $\mathrm{n}=138$, Age: $29.5(5.3)$ & $\mathrm{n}=144$, Age: $28.2(5.4)$ & PIH:13.8 vs. 13.2; Labor induction: 26.8 vs. 26.4 \\
\hline Waters et al., 2016 & $\begin{array}{l}\text { North } \\
\text { Ameri- } \\
\text { can }\end{array}$ & $\begin{array}{l}\text { (1) IADPSG } \\
\text { (2) CC }\end{array}$ & 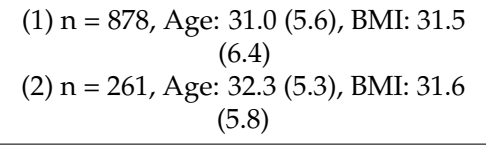 & $\mathrm{n}=5020$, Age: 30.1 (5.8), BMI: 28.2 & $\begin{array}{c}\text { (1) Preeclampsia: } 14.9 \text { vs. } 6.4 \text {; Primary cesarean section: } 23.9 \text { vs. } \\
17.2 \\
\text { (2) Preeclampsia: } 14 \text { vs. } 6.4 \text {; Primary cesarean section: } 30.4 \text { vs. } \\
17.2\end{array}$ \\
\hline Gu et al., 2019 & China & WHO-1999 & $\begin{array}{c}\text { GDM with hypertensive disorders of } \\
\text { pregnancy: } \\
\text { n = 91, Age: } 33.8 \text { (3.59), } \\
\text { Pre-pregnancy BMI: } 25.1(3.64) \\
\text { GDM without hypertensive disorders } \\
\text { of pregnancy: } \\
\text { n = 1172, Age: } 33.3(3.49), \\
\text { Pre-pregnancy BMI: } 22.9(3.24)\end{array}$ & $\begin{array}{c}\text { Non-GDM with hypertensive } \\
\text { disorders of pregnancy: } \\
\mathrm{n}=261 \text {, Age: } 32.9(2.68), \\
\text { Pre-pregnancy BMI: } 22.2(3.04) \\
\text { Non-GDM without hypertensive } \\
\text { disorders of pregnancy: } \\
\mathrm{n}=261 \text {, Age: } 32.9(2.84), \\
\text { Pre-pregnancy BMI: } 21.4(2.96)\end{array}$ & $\begin{array}{c}\text { Non hypertensive disorder: Gestational weight gain, kg: } 16.6 \\
\text { (5.87) vs. } 18.2 \text { (6.67) } \\
\text { Hypertensive disorder: Gestational weight gain, kg: } 19.0 \text { (7.01) } \\
\text { vs. } 21.3(6.14)\end{array}$ \\
\hline Anderberg et al., 2010 & Sweden & WHO-1999 & $\mathrm{n}=306$, Age: $32(18-46)$ & $\mathrm{n}=329$, Age: $31(20-42)$ & Labor induction: 18.6 vs. 6.4 \\
\hline Avalos et al., 2013 & Ireland & IADPSG & $\mathrm{n}=622$, Age: 32.8 & $\mathrm{n}=4225$, Age: 31 (4.9) & $\begin{array}{l}\text { GDM without risk factor vs. GDM with risk factor vs. Non-GDM } \\
\text { Hypertension: } 13 \text { vs. } 15 \text { vs. } 7\end{array}$ \\
\hline Wahabi et al., 2017 & $\begin{array}{l}\text { Saudi } \\
\text { Arabia }\end{array}$ & WHO-2013 & $\mathrm{n}=2354$, Age: $31.5(5.9)$ & $\mathrm{n}=6951$, Age: $29.5(5.7)$ & $\begin{array}{c}\text { Gestational hypertension: } 1.8 \text { vs. } 1.3 ; \\
\text { Preeclampsia/superimposed: } 1 \text { vs. } 1.1 \text {; Labor induction: } 17.9 \text { vs. } \\
16\end{array}$ \\
\hline Meek et al., 2015 & UK & $\begin{array}{l}\text { (1) IADPSG } \\
\text { (2) NICE }\end{array}$ & $\begin{array}{l}\text { (1) } \mathrm{n}=387, \text { Age: } 32.6, \text { BMI: } 27.4 \\
\text { (2) } \mathrm{n}=261 \text {, Age: } 32.1, \text { BMI: } 25.5\end{array}$ & $\mathrm{n}=2406$, Age: 31.4, BMI: 26 & $\begin{array}{l}\text { (1) Pre-eclampsia: } 10.1 \text { vs. } 7.2 \text {; PPH:1 vs. } 2 \text {; APH: } 1.6 \text { vs. } 2.4 \\
\text { (2) Pre-eclampsia: } 9.2 \text { vs. } 7.2 \text {;PH:0.4 vs. 2; APH: } 2.7 \text { vs. } 2.4\end{array}$ \\
\hline Boghossian et al., 2014 & USA & ICD-9 & $\begin{array}{c}\mathrm{n}=1279, \text { Age: } 30.3(4.9) ; \\
\text { Prepregnancy BMI: } 28.9(7.2)\end{array}$ & $\begin{array}{c}\mathrm{n}=58,224, \text { Age: } 28.1(4.5) \\
\text { Prepregnancy BMI: } 24.9(5.6)\end{array}$ & $\begin{array}{l}\text { Gestational hypertension: } 4.7 \text { vs. } 2.2 \text {; Preeclampsia: } 3 \text { vs. } 1.6 \text {; } \\
\text { Labor induction: } 40.2 \text { vs. } 39.4\end{array}$ \\
\hline Kawakita et al., 2017 & USA & ICD-9 & $\begin{array}{c}\mathrm{n}=11,327, \text { Age: } 30.8(6.0), \text { BMI: } 34.1 \\
(7.5)\end{array}$ & $\begin{array}{c}\mathrm{n}=208,355, \text { Age: } 27.4 \text { (6.1), BMI: } 30.6 \\
(6.1)\end{array}$ & Pregnancy-associated hypertension: 11.7 vs. 7.2 \\
\hline
\end{tabular}


Table 1. Cont.

\begin{tabular}{|c|c|c|c|c|c|}
\hline Author, Year & Country & $\begin{array}{c}\text { GDM } \\
\text { Diagnostic Criteria }\end{array}$ & $\begin{array}{c}\text { GDM } \\
\text { Characteristics * }\end{array}$ & Non-GDM Characteristics * & $\begin{array}{l}\text { Adverse Maternal Outcome in Women with vs. without } \\
\text { GDM, \% or Mean (SD) }\end{array}$ \\
\hline Brand et al.2018 & UK & Modified WHO-1999 & $\begin{array}{l}\text { White European: } \mathrm{n}=210, \text { Age: } 30.2 \\
\text { (5.4), BMI: } 28.6 \text { (6.3) } \\
\text { South Asian: } \mathrm{n}=622 \text {, Age: } 30.7 \text { (5.3), } \\
\text { BMI: } 28.2 \text { (5.8) }\end{array}$ & $\begin{array}{l}\text { White European: } \mathrm{n}=4537 \text {, Age: } 26.6 \\
\text { (6.0), BMI: } 26.5 \text { (5.9) } \\
\text { South Asian: } \mathrm{n}=5336 \text {, Age: } 27.7 \text { (5.0), } \\
\text { BMI: } 25.2 \text { (5.3) }\end{array}$ & 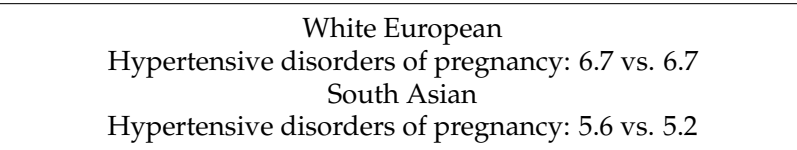 \\
\hline Kaul et al., 2014 & Canada & CDA-2013 & $\begin{array}{l}\text { GDM only: } \mathrm{n}=7332 \text {, Age: } 31.9(5.5) \\
\text { GDM and overweight: } \mathrm{n}=1399 \text {, Age: } \\
31 \text { (5.2) }\end{array}$ & $\mathrm{n}=213,765$, Age: $28.6(5.6)$ & $\begin{array}{l}\text { GDM only vs. GDM and overweight vs. No GDM, not } \\
\text { overweight } \\
\text { Pre-eclampsia or eclampsia: } 1.9 \text { vs. } 5.5 \text { vs. } 1.2 \text {; Labor induction: } \\
42.1 \text { vs. } 58.4 \text { vs. } 28.5\end{array}$ \\
\hline Kgosidialwa et al., 2015 & Ireland & IADPSG & $\mathrm{n}=567$, Age: 33.4 (4.9), BMI: 30.5 (6.1) & $\mathrm{n}=2499$, Age: 31.5 (5.2), BMI: 26.7 & $\begin{array}{c}\text { Pre-eclampsia: } 4.2 \text { vs. } 3.8 \text {; Hypertensive pregnancy disorders: } \\
\text { 11.6 vs. } 8.3 \\
\text { PIH: } 11.6 \text { vs. } 7.7\end{array}$ \\
\hline Donovan et al., 2017 & Canada & $\begin{array}{l}\text { CDA } \\
\text { IADPSG }\end{array}$ & $\begin{array}{c}\text { HAPO 1.75: } \\
\mathrm{n}=\text { 4308, Age: } 31.2(5.1) \\
\text { HAPO 2- } 1: \\
\mathrm{n}=5528, \text { Age: } 31.6(5.2) \\
\text { HAPO 2-2: } \\
\mathrm{n}=3252, \text { Age: } 32.1(5.2)\end{array}$ & $\begin{array}{l}\text { Normal } 50 \text { g screen: } \\
\mathrm{n}=144,191, \text { Age: } 28.8(5.3) \\
\text { Normal } 75 \text { g OGTT: } \\
\mathrm{n}=21,248, \text { Age: } 30.3(5.3)\end{array}$ & $\begin{array}{l}\text { Normal } 50 \mathrm{~g} \text { screen: } \\
\text { Hypertensive disorders of pregnancy: } 5.6 \text {; Labor induction: } 27.5 \\
\quad \text { Normal } 75 \mathrm{~g} \text { OGTT: } \\
\text { Hypertensive disorders of pregnancy: } 7.3 \text {; Labor induction: } 27.7 \\
\text { HAPO 1.75: } \\
\text { Hypertensive disorders of pregnancy: } 9.1 \text {; Labor induction: } 29.6 \\
\text { HAPO 2-1: } \\
\text { Hypertensive disorders of pregnancy: } 9.6 \text {; Labor induction: } 38.2 \\
\text { HAPO 2-2: } \\
\text { Hypertensive disorders of pregnancy: } 11.7 \text {; Labor induction: } 42.3\end{array}$ \\
\hline Kieffer et al., 1999 & Michigan & NDDG & $\mathrm{n}=19$, Age: 29.4 (6.2), BMI: 28.7 (5.7) & $\begin{array}{c}\mathrm{n}=353, \text { Age: } 24.79(4.85), \text { BMI: } 25.1 \\
(4.21)\end{array}$ & Hypertensive disorder: 21.1 vs. 7.16 \\
\hline Ekeroma et al., 2014 & $\begin{array}{l}\text { New } \\
\text { Zealand }\end{array}$ & $\begin{array}{l}\text { (1) NZSSD } \\
\text { (2) IADPSG } \\
\text { (3) ADIPS }\end{array}$ & $\begin{array}{c}\text { (1) } \mathrm{n}=381 \text {, Age: } 31.7 \text { (5.5), BMI: } 31.8 \\
(10.8) \\
\begin{array}{c}\text { (2) } \mathrm{n}=238, \text { Age: } 31.4(5.8), \text { BMI: } 32.9 \\
(11.7) \\
\text { (3) } \mathrm{n}=608, \text { Age: } 31.5(5.4), \text { BMI: } 30.5 \\
(9.8)\end{array}\end{array}$ & $\begin{array}{c}\mathrm{n}=1672, \text { Age: } 30.0(5.7), \text { BMI: } 30.7 \\
(9.1)\end{array}$ & $\begin{array}{l}\text { (1) Pre-eclampsia: } 8 \text { vs. } 6 \\
\text { (2) Pre-eclampsia: } 7 \text { vs. } 6 \\
\text { (3) Pre-eclampsia: } 7 \text { vs. } 6\end{array}$ \\
\hline Aung et al., 2015 & $\begin{array}{l}\text { Cook } \\
\text { Islands }\end{array}$ & Modified IADPSG & $\begin{array}{c}\mathrm{n}=94, \text { Age: } 36(28-40), \text { BMI: } 34 \\
(30-39)\end{array}$ & $\begin{array}{c}\mathrm{n}=28(23-34), \text { Age: } 24.79 \text { (4.85), BMI: } \\
31(26-36)\end{array}$ & Pregnancy weight gain (kg): 6 (3-11) vs. 10 (6-14) \\
\hline Erjavec et al., 2016 & Croatia & $\begin{array}{l}\text { (1) WHO-1999 } \\
\text { (2) IADPSG }\end{array}$ & $\begin{array}{l}\text { (1) } \mathrm{n}=953, \text { Age: } 30.88 \text { (5.23), BMI: } \\
25.84(5.28) \\
\text { (2) } \mathrm{n}=1829 \text {, Age: } 31.34 \text { (5.19), BMI: } \\
26.03(5.64)\end{array}$ & $\begin{array}{c}\text { (1) } \mathrm{n}=41,703 \text {, Age: } 28.77 \text { (5.23), BMI: } \\
\text { 23.38 (3.99) } \\
\text { (2) } \mathrm{n}=37,263 \text {, Age: } 29.49 \text { (5.33), BMI: } \\
\text { 23.38 (4.11) }\end{array}$ & $\begin{array}{l}\text { (1) Weight gain: } 12.57(5.62) \text { vs. } 14.51(5.29) \\
\text { (2) Weight gain: } 12.50(5.76) \text { vs. } 14.19(5.71)\end{array}$ \\
\hline Gortazar et al., 2018 & Spain & NDDG & $\mathrm{n}=35,729$, Age: 33.42 & $\mathrm{n}=704,148$, Age: 31.27 & Pre-eclampsia: 2.56 vs. 1.44 \\
\hline Zamstein et al., 2018 & Israel & ACOG & $\begin{array}{l}\text { GDM A1: } \mathrm{n}=9460, \text { Age: } 32.1(5.8) \\
\text { GDM A2: } \mathrm{n}=724, \text { Age: } 33.7(5.6)\end{array}$ & $\mathrm{n}=206,013$, Age: $28(5.7)$ & $\begin{array}{c}\text { GDM A1 vs. GDM A2 vs. Non-GDM } \\
\text { Hypertensive disorders of pregnancy: } 11.2 \text { vs. } 18.1 \text { vs. } 4.8\end{array}$ \\
\hline
\end{tabular}


Table 1. Cont.

\begin{tabular}{|c|c|c|c|c|c|}
\hline Author, Year & Country & $\begin{array}{c}\text { GDM } \\
\text { Diagnostic Criteria }\end{array}$ & $\begin{array}{c}\text { GDM } \\
\text { Characteristics * }\end{array}$ & Non-GDM Characteristics * & $\begin{array}{l}\text { Adverse Maternal Outcome in Women with vs. without } \\
\text { GDM, \% or Mean (SD) }\end{array}$ \\
\hline Hedderson et al., 2003 & California & $\begin{array}{l}\text { (1) NDDG } \\
\text { (2) CC }\end{array}$ & $\begin{array}{l}(1) n=1523 \\
(2) n=840\end{array}$ & $\mathrm{n}=38,515$ & $\begin{array}{l}\text { (1) Pregnancy-induced hypertension: } 3.4 \text { vs. } 1.9 \text {; Preeclampsia or } \\
\text { eclampsia:5.8 vs. } 2.9 \text {; Placenta previa: } 0.6 \text { vs. } 0.1 \text {; Abruptio } \\
\text { placentae: } 1 \text { vs. } 0.8 \text {; Labor induction: } 18.4 \text { vs. } 14.5 \\
\text { (2) Pregnancy-induced hypertension: } 3.6 \text { vs. } 1.9 \text {; Preeclampsia or } \\
\text { eclampsia: } 5.6 \text { vs. } 2.9 \text {; Placenta previa: } 0.8 \text { vs. } 0.1 \text {; Abruptio } \\
\text { placentae: } 0.5 \text { vs. } 0.8 \text {; Labor induction: } 13.5 \text { vs. } 14.5\end{array}$ \\
\hline Hosseini et al., 2018 & Iran & IADPSG & $\begin{array}{c}\text { Early-onset GDM: } \\
\mathrm{n}=93 \text {, Age: } 30.7 \text { (4.6), Pre-pregnancy } \\
\text { BMI: } 26.5(4.2) \\
\text { Late-onset GDM: } \\
\mathrm{n}=78 \text {, Age: } 31.1 \text { (4.9), Pre-pregnancy } \\
\text { BMI: } 26.2(4.7)\end{array}$ & $\begin{array}{c}\mathrm{n}=758, \text { Age: } 28.8(4.6) \\
\text { Pre-pregnancy BMI: } 24.2(4.1)\end{array}$ & $\begin{array}{l}\text { Early-onset GDM vs. Late-onset GDM vs. Normal } \\
\text { Preeclampsia: } 6.5 \text { vs. } 6.4 \text { vs. } 3.6 \\
\text { Gestational hypertension: } 8.6 \text { vs. } 12.8 \text { vs. } 6.1\end{array}$ \\
\hline Hosseini et al., 2018 & Iran & $\begin{array}{l}\text { (1) IADPSG } \\
\text { (2) CC }\end{array}$ & $\begin{array}{l}\text { (1) } n=78, \text { Age: } 18-45 \\
\text { (2) } n=35 \text {, Age: } 18-45\end{array}$ & $\begin{array}{l}\text { (1) } \mathrm{n}=35 \text {, Age: } 18-45 \\
\text { (2) } \mathrm{n}=801 \text {, Age: } 18-45\end{array}$ & $\begin{array}{l}\text { (1) Preeclampsia (OR): } 1.5 \text {; Gestational hypertension (OR): } 1.9 \\
\text { (2) Preeclampsia (OR): 2.8; Gestational hypertension (OR): } 2.4\end{array}$ \\
\hline Jain et al., 2016 & India & DIPSI & $\mathrm{N}=8000$ & $\mathrm{n}=7641$ & PIH: 9 vs. 6 ; APH/PPH: 0.84 vs. 0.32 \\
\hline Kun et al., 2010 & Tolna & WHO-1999 & $\begin{array}{l}\mathrm{n}=139, \text { Age: } 29.6 \text { (5.2), Pregnancy } \\
\text { BMI: } 25.4(5.3)\end{array}$ & $\begin{array}{c}\mathrm{n}=2583 \text {, Age: } 27.1(4.9), \text { Pregnancy } \\
\text { BMI: } 23.1(4.5)\end{array}$ & Weight gain, kg: 9.1 (4.8) vs. 12.9 (5.0) \\
\hline Jacobson et al., 1989 & California & NDDG & n = 97, Age: 28.8 (0.5), BMI: 27.6 (0.8) & $\begin{array}{c}\mathrm{n}=2107, \text { Age: } 26.3(0.1), \text { BMI: } 22.8 \\
(0.1)\end{array}$ & $\begin{array}{c}\text { Pregnancy-induced hypertension: } 3.8 \text { vs. 3.7; Weight gain: } 30.2 \\
\text { (1.8) (pounds) vs. } 33.0(0.3)\end{array}$ \\
\hline Pan et al., 2015 & China & $\begin{array}{l}\text { (1) WHO-1999 } \\
\text { (2) IADPSG }\end{array}$ & $\begin{array}{c}\text { (1) } \mathrm{n}=257, \text { Age: } 29(2.6) \\
\text { Prepregnancy BMI: } 22.9(3.5) \\
\text { (2) } \mathrm{n}=429 \text {, Age: } 28.8(2.9), \\
\text { Prepregnancy BMI: } 23.9(4)\end{array}$ & $\begin{array}{c}\mathrm{n}=16 \text { 173, Age: } 28.4(2.8) \\
\text { Prepregnancy BMI: } 22.1(3.3)\end{array}$ & $\begin{array}{l}\text { (1) PIH: } 15.8 \text { vs. } 4.8 \\
\text { (2) PIH: } 7.5 \text { vs. } 4.8\end{array}$ \\
\hline Son et al., 2014 & Korea & ICD-10 & $\mathrm{n}=78,716$, Age: $15-49$ & $\mathrm{n}=1171575$, Age: $15-49$ & $\begin{array}{c}\text { Pregnancy-induced hypertension without significant proteinuria: } \\
1.71 \text { vs. } 1 \text {; Pregnancy-induced hypertension with significant } \\
\text { proteinuria: } 1.66 \text { vs. } 1.13 \text {; Eclampsia: } 0.08 \text { vs. } 0.05 \text {; Placenta } \\
\text { previa: } 1.41 \text { vs. } 1.16 \text {; Premature separation of placenta: } 0.42 \text { vs. } \\
0.42 \text {; Postpartum hemorrhage: } 7.03 \text { vs. } 7.30 \text {; Antepartum } \\
\text { hemorrhage: } 2.29 \text { vs. } 2.39\end{array}$ \\
\hline Katterfeld et al., 2011 & Australia & ADIPS & $\begin{array}{l}\text { Australian born } \\
\mathrm{n}=4765 \\
\text { CALD } \\
\mathrm{n}=1686 \\
\text { Non-CALD } \\
\mathrm{n}=1273\end{array}$ & $\begin{array}{l}\text { Australian born } \\
\mathrm{n}=142,537 \\
\text { CALD } \\
\mathrm{n}=23,541 \\
\text { Non-CALD } \\
\mathrm{n}=31,814\end{array}$ & $\begin{array}{c}\text { Australian born } \\
\text { Pre-eclampsia: } 8.4 \text { vs. 5; Labor induction: } 54.3 \text { vs. } 37.3 \\
\text { CALD } \\
\text { Pre-eclampsia: } 5.6 \text { vs. 3.6; Labor induction: } 37.6 \text { vs. } 25.7 \\
\text { Non-CALD } \\
\text { Pre-eclampsia: } 7.2 \text { vs. } 4.6 \text {; Labor induction: } 51.9 \text { vs. } 35\end{array}$ \\
\hline
\end{tabular}


Table 1. Cont.

\begin{tabular}{|c|c|c|c|c|c|}
\hline Author, Year & Country & $\begin{array}{c}\text { GDM } \\
\text { Diagnostic Criteria }\end{array}$ & $\begin{array}{c}\text { GDM } \\
\text { Characteristics * }\end{array}$ & Non-GDM Characteristics * & $\begin{array}{l}\text { Adverse Maternal Outcome in Women with vs. without } \\
\text { GDM, \% or Mean (SD) }\end{array}$ \\
\hline Sacks et al., 2015 & California & IADPSG & $\begin{array}{l}\text { (1) GDM-1: } \mathrm{n}=771 \text {, Age: } 30.9(5.6) \\
\text { (2) GDM-2: } \mathrm{n}=1121 \text {, Age: } 31 \text { (5.7) }\end{array}$ & $\mathrm{n}=7943$, Age: $26.3(0.1)$ & $\begin{array}{c}\text { GDM-1 vs. GDM-2 vs. normal } \\
\text { Preeclampsia-eclampsia: } 4.3 \text { vs. } 7.7 \text { vs. } 4.4 \\
\text { Primary cesarean delivery: } 20.6 \text { vs. } 22.3 \text { vs. } 16.6\end{array}$ \\
\hline Soliman et al., 2018 & Qatar & IADPSG & $\mathrm{n}=3027$ & $\mathrm{n}=8995$ & Hypertensive disorders: 5.5 vs. 3.5 ; Labor induction: 26.5 vs. 12.4 \\
\hline Xiong et al., 2001 & Canada & $\mathrm{CDA}$ & $\mathrm{n}=2755$ & $\mathrm{n}=8995$ & Gestational hypertension: 11.4 vs. 4.8 ; Pre-eclampsia: 1.1 vs. 1.1 \\
\hline Oster et al., 2014 & Canada & CDA & $\mathrm{n}=1224$, Age: $28.8(6.27)$ & $\mathrm{n}=26,793$, Age: $24.7(5.8)$ & $\begin{array}{l}\text { Pregnancy induced hypertension: } 11.3 \text { vs. } 4.4 \text {; Labor induction: } \\
43.6 \text { vs. } 23.8\end{array}$ \\
\hline Sugaya et al., 2000 & Japan & $\begin{array}{l}\text { (1) JSOG } \\
\text { (2) WHO-1998 }\end{array}$ & $\begin{aligned} & \text { (1) } \mathrm{n}=55 \text {, Age: } 29.7 \text { (4.3), BMI: } 26.2 \\
&(3.4) \\
& \text { (2) } \mathrm{n}=51 \text {, Age: } 32.8(4.3), \text { BMI: } 26.5 \\
&(4.3)\end{aligned}$ & $\mathrm{n}=281$, Age: 30 (4.7), BMI: 25.5 (3.3) & $\begin{array}{l}\text { (1) preeclampsia: } 18 \text { vs. } 17 \\
\text { (2) preeclampsia: } 28 \text { vs. } 17\end{array}$ \\
\hline Nerenberg et al., 2013 & Canada & $\mathrm{CDA}$ & $\mathrm{n}=15,404$, Age: $31.5(5.4)$ & $\mathrm{n}=407,268$, Age: $28.4(5.6)$ & $\begin{array}{l}\text { Preeclampsia/eclampsia: } 2.6 \text { vs. 1.2; Labor induction: } 41.9 \text { vs. } \\
27.1\end{array}$ \\
\hline Edith Kieffer et al., 2006 & Mexico & ADA-2003 & $\mathrm{n}=68$, Age: 28.6 (0.6), BMI: $25.7(0.2)$ & $\mathrm{n}=933$, Age: $24.8(0.2)$, BMI: $28.4(0.8)$ & Weight gain $(\mathrm{kg}): 10.0(0.6)$ vs. $13(0.2)$ \\
\hline $\begin{array}{l}\text { Goswami Mahanta et al., } \\
\qquad 2014\end{array}$ & India & DIPSI & $\mathrm{N}=28$ & $\mathrm{n}=749$ & Gestational hypertension: 53.6 vs. 28.1 \\
\hline Sletner et al., 2017 & Norway & WHO-1999 & $\begin{array}{c}\text { Europe } \\
\text { Mild: } \mathrm{n}=30 \text {, Age: } 31.2(29.5), \text { BMI: } \\
\text { 25.5 }(23.8,27.2) \\
\text { Moderate/severe: } \mathrm{n}=9 \text {, Age: } 30.6 \\
(27.6,33.5) \text {, BMI: } 30.5(27.4,33.6) \\
\text { South Asia } \\
\text { Mild: } \mathrm{n}=9 \text {, Age: } 30.7(28.3,33.0) \\
\text { BMI: } 25.3(23.2,27.5) \\
\text { Moderate/severe: } \mathrm{n}=4724, \text { Age: } 30.4 \\
(28.0,32.7), \text { BMI: } 22.7(20.6,24.9)\end{array}$ & $\begin{array}{c}\text { Europe } \\
\mathrm{n}=310 \text {, Age: } 30.6(30.1,31.1), \mathrm{BMI}: \\
24.3(23.8,24.8) \\
\text { South Asia } \\
\mathrm{n}=156 \text {, Age: } 28.4(27.7,29.1), \text { BMI: } \\
23.7(23.0,24.3)\end{array}$ & $\begin{array}{c}\text { Europe } \\
\text { Mild vs. Moderate/Severe vs. Non-GDM } \\
\text { Mild hypertension/preeclampsia: } 10 \text { vs. } 0 \text { vs. } 7 \text {; Severe } \\
\text { hypertension/ preeclampsia: } 2 \text { vs. } 0 \text { vs. } 2 \text {; inclusion to week } 28 \\
\text { GWG: } 6.2(5.2,7.2) \text { vs. } 5.2(3.4,7.1) \text { vs. } 7.1(6.8,7.4) \text {, week } 28 \text { to } \\
\text { birth: } 4.0(2.6,5.5) \text { vs. } 2.0(-0.4,4.4) \text { vs. } 5.9(5.5,6.4) \\
\text { South Asia } \\
\text { Mild hypertension/preeclampsia: } 7 \text { vs. } 14 \text { vs. } 3 \text {; Severe } \\
\text { hypertension/ preeclampsia: } 0 \text { vs. } 7 \text { vs. } 2 \text {; inclusion to week } 28 \text {, } \\
\text { GWG: } 5.6(3.9,7.4) \text { vs. } 6.5(4.7,8.2) \text { vs. } 6.6(6.0,7.1) \text {, week } 28 \text { to } \\
\text { birth, GWG: } 5.1(2.9,7.4) \text { vs. } 4.8(2.5,7.0) \text { vs. } 5.2(4.5,5.9)\end{array}$ \\
\hline Zeki et al., 2018 & Australia & ADIPS & $\mathrm{n}=51135$, Age: $32.2(5.3)$ & $\mathrm{n}=950$ 678, Age: 29.9 (5.6) & Primary Cesarean: Relative \% 13.8 vs. 13.5 \\
\hline Hoorn et al., 2002 & Australia & ADIPS & n = 51, Age: 30.9 (5.7), BMI:31.5 (.1) & $\mathrm{n}=258$, Age: 24.9 (6.3), BMI: 25.5 (5.9) & $\begin{array}{l}\text { Gestational hypertension: } 45.1 \text { vs. 29.1; Preeclampsia: } 19.6 \text { vs. } \\
17.1\end{array}$ \\
\hline
\end{tabular}


Table 1. Cont.

\begin{tabular}{|c|c|c|c|c|c|}
\hline Author, Year & Country & $\begin{array}{c}\text { GDM } \\
\text { Diagnostic Criteria }\end{array}$ & $\begin{array}{c}\text { GDM } \\
\text { Characteristics * }\end{array}$ & Non-GDM Characteristics * & $\begin{array}{l}\text { Adverse Maternal Outcome in Women with vs. without } \\
\text { GDM, \% or Mean (SD) }\end{array}$ \\
\hline Su et al., 2019 & China & China National criteria & $\begin{array}{c}\text { Underweight } \\
\mathrm{n}=1466, \text { BMI: } 17.55(0.79) \\
\text { Normal weight } \\
\mathrm{n}=6905, \text { BMI: } 20.80(1.21) \\
\text { Overweight } \\
\mathrm{n}=2220, \text { BMI: } 23.86(0.57) \\
\text { Obese } \\
\mathrm{n}=2252, \text { BMI: } 27.21(2.15)\end{array}$ & $\begin{array}{c}\text { Underweight } \\
\mathrm{n}=12,336, \text { BMI: } 17.54(0.79) \\
\text { Normal weight } \\
\mathrm{n}=36,935, \text { BMI: } 20.54(1.2) \\
\text { Overweight } \\
\mathrm{n}=6654, \text { BMI: } 23.82(0.56) \\
\text { Obese } \\
\mathrm{n}=4730, \text { BMI: } 26.97(1.97)\end{array}$ & $\begin{array}{c}\text { Normal weight } \\
\text { weight gain, kg: } 11.45 \text { (3.98) vs. } 13.15(0.25) \\
\text { Underweight } \\
\text { weight gain, kg: } 12.53(3.94) \text { vs. } 13.76(3.93) \\
\text { Overweight } \\
\text { weight gain, kg: } 10.92(4.49) \text { vs. } 12.29(4.48) \\
\text { Obese } \\
\text { weight gain, kg: } 8.87(4.38) \text { vs. } 10.50(4.35)\end{array}$ \\
\hline Carr et al., 2011 & USA & ICD-9\&10 & n = 1314, Age: 32.7 (5.7) & $\begin{array}{c}\text { One abnormal: } \mathrm{n}=1242 \text {, Age: } 32.3 \\
(5.3) \\
\text { Non abnormal: } \mathrm{n}=3620 \text {, Age: } 32(5.7)\end{array}$ & Preeclampsia (n): 111 vs. 102 vs. 226 \\
\hline Lamminpää et al., 2014 & Finland & ICD-10 & $\begin{array}{l}<35 \mathrm{y}: \mathrm{n}=19,422 \\
>35 \mathrm{y}: \mathrm{n}=7732\end{array}$ & $\begin{array}{c}<35 \mathrm{y}: \mathrm{n}=210,581 \\
>35 \mathrm{y}: \mathrm{n}=45,589.00\end{array}$ & $\begin{array}{c}\text { <35 y: Normal glucose tol. vs. Diet-treated vs. Insulin-treated } \\
\text { Preeclampsia: } 4.2 \text { vs. } 6.7 \text { vs. } 7.7 \text {; Placenta previa: } 0.2 \text { vs. } 0.2 \text { vs. } \\
0.2 \\
\text { Late pregnancy bleeding: } 1 \text { vs. } 1.2 \text { vs. } 1.8 \\
>35 \text { y: Normal glucose tol. vs. Diet-treated vs. Insulin-treated } \\
\text { Preeclampsia: } 5.1 \text { vs. } 8.2 \text { vs. } 8.6 \text {; Placenta previa: } 0.4 \text { vs. } 0.5 \text { vs. } \\
\text { 0.1; Late pregnancy bleeding: } 1.3 \text { vs. } 1.3 \text { vs. } 1.4\end{array}$ \\
\hline Black et al., 2010 & California & IADPSG & $\begin{array}{c}\text { single isolated impaired glucose } \\
\text { tolerance (i-IGT1) } \\
\mathrm{n}=391 \text {, Age: } 32.1 \text { (5.4), BMI: } 28.1 \text { (5.6) } \\
\text { isolated impaired fasting glucose } \\
\text { (i-IFG) } \\
\mathrm{n}=886 \text {, Age: } 30.4 \text { (5.6), BMI: } 30.8 \text { (7.1) } \\
\text { double-isolated impaired glucose } \\
\text { tolerance (i-IGT2) } \\
\mathrm{n}=83 \text {, Age: } 32.3 \text { (5.2), BMI: } 27.5 \text { (4.7) } \\
\text { IFG + IGT } \\
\mathrm{n}=331 \text {, Age: } 32 \text { (5.1), BMI: } 31.8 \text { (7) }\end{array}$ & $\begin{array}{c}\mathrm{n}=7020, \text { Age: } 28.6(5.9), \text { BMI: } 26.9 \\
(5.8)\end{array}$ & $\begin{array}{c}\text { i-IGT1 vs. i-IFG vs. i-IGT2 vs. IFG + IGT vs. No GDM } \\
\text { Gestational hypertension: } 9.8 \text { vs. } 10.8 \text { vs. } 13.6 \text { vs. } 15.4 \text { vs. } 7.2 \text {; } \\
\text { Primary cesarean section: } 12.8 \text { vs. } 9.1 \text { vs. } 18.1 \text { vs. } 8.2 \text { vs. } 6.6 \text {; } \\
\text { gestational weight gain: } 119(30.4) \text { (Ib) vs. } 427(48.2) \text { vs. } 23(27.7) \\
\text { vs. } 175(52.9) \text { vs. } 1737(24.7)\end{array}$ \\
\hline
\end{tabular}

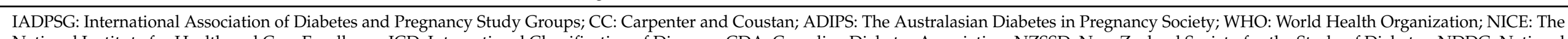

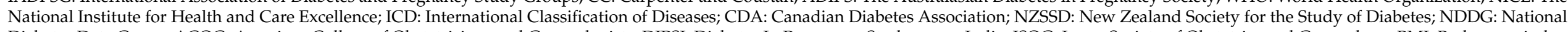

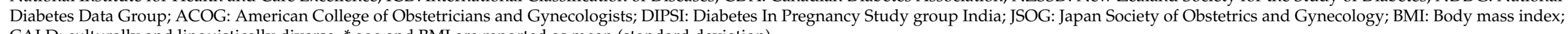
CALD: culturally and linguistically diverse. * age and BMI are reported as mean (standard deviation). 
The Supplementary Tables S1 and S2 contain the results of quality assessment. All studies were categorized as high quality [20-68]. A total of 95.9\% studies were prospective or retrospective cohorts [22-68] and 4\% were cross-sectional studies [20,21]. In addition, 17 $(34.6 \%)$ studies used the GDM classification of group $1[21,22,26,35,38,40,47,48,50,51,53,54$, 59-61,64,67] and IADPSG; 7 (14.2\%) group 2 [20,27,28,51,59,65,68], 3 (6.1\%) group 3 [32,46, 56], 1 (2\%) group 4 [51], 19 (38.7\%) group 5 [23-25,29,31,33,34,36,39,40,42,44,47,53,55,57,58, 64,66], 6 (12.2\%) group 6 [37,41,43,44,49,52] and 6 (12.2\%) group 7 [21,29,30,45,62,63].

It should be noted that 9 studies used more than one GDM classification $[21,29,40,44$, 47,51,53,59,64] as follows: 4 studies used classifications 1 and 5 [40,47,52,63], one used 1 and 2 classifications [59], one used classifications 1, 2 and 4 [51], one used classifications 1 and 7 [21], one used classifications 5 and 6 [44], and finally one used classifications 5 and 7 [29].

In addition, $34.69 \%$ of the studies were conducted in the U.S. [22,24,25,31,33,34,36$38,41,44,52,53,55,57,58,64], 14.2 \%$ in Australia [20,27,28,50,51,65,68], 28.5\% in Asia [26,29, $32,35,39,40,42,46-49,60,63,66]$, and 22.4\% in Europe [21,23,30,43,45,54,56,59,61,62,67].

\subsection{Meta-Analysis and Meta-Regression Results}

The overall pooled $\mathrm{OR} /$ mean difference $(95 \% \mathrm{CI})$ of adverse maternal outcomes, its heterogeneity, and the estimation of publication bias among various subgroups of GDM diagnosis criteria, compared to non-GDM counterparts have been presented in Table 2.

Table 2. Results of meta-analyses for risk/standardized mean difference adverse maternal outcome among women with gestational diabetes according to different GDM screening strategy group.

\begin{tabular}{|c|c|c|c|c|c|c|c|c|}
\hline \multirow[b]{2}{*}{ Outcomes $^{£}$} & \multirow[b]{2}{*}{$\begin{array}{c}\text { GDM } \\
\text { Classification }\end{array}$} & \multicolumn{2}{|c|}{ Sample Size } & \multicolumn{2}{|c|}{ Heterogenicity } & \multirow[b]{2}{*}{$\begin{array}{c}\text { Publication } \\
\text { Bias Begg's } \\
\text { Test }\end{array}$} & \multirow[b]{2}{*}{$\begin{array}{l}\text { Effect Size * } \\
(95 \% \mathrm{CI})\end{array}$} & \multirow{2}{*}{$\begin{array}{c}p \text {-Value } \\
\text { from Meta- } \\
\text { Regression }\end{array}$} \\
\hline & & $\begin{array}{l}\text { GDM } \\
\text { Group }\end{array}$ & $\begin{array}{l}\text { Non- } \\
\text { GDM } \\
\text { Group }\end{array}$ & $I^{2}(\%)$ & $p$-Value & & & \\
\hline Primary & 1 & 4632 & 49,353 & 21.1 & 0.262 & 0.621 & $1.3(1.2,1.5)$ & Ref \\
\hline \multirow[t]{2}{*}{ Cesarean } & Overall & 4990 & 56,480 & 41 & 0.084 & 0.655 & $1.4(1.2,1.5)$ & - \\
\hline & 1 & 10,098 & 183,424 & 95.2 & 0.001 & 0.327 & $1.3(0.9,1.8)$ & Ref \\
\hline \multirow{4}{*}{$\begin{array}{l}\text { Induction of } \\
\text { labor }\end{array}$} & 2 & 25,197 & 549,639 & 94.7 & 0.001 & 0.851 & $1.8(1.5,2.1)$ & 0.144 \\
\hline & 5 & 196,263 & $4,151,466$ & 97.4 & 0.001 & 0.371 & $1.8(1.6,2.0)$ & 0.112 \\
\hline & Overall & 233,767 & $4,925,044$ & 97.5 & 0.001 & 0.766 & $1.7(1.6,1.9)$ & - \\
\hline & 2 & 67,430 & $1,404,544$ & 79.9 & 0.001 & 0.348 & $1.2(1.0,1.4)$ & ${ }^{€}$ Ref \\
\hline Maternal & 5 & 609,575 & $9,821,846$ & 95 & 0.001 & 0.680 & $1.1(1.0,1.3)$ & 0.867 \\
\hline \multirow[t]{4}{*}{ Hemorrhage } & 6 & 3046 & 77,031 & 91.9 & 0.001 & 0.317 & $2.6(0.5,12.6)$ & 0.126 \\
\hline & Overall & 688,825 & $11,315,874$ & 93 & 0.001 & 0.523 & $1.2(1.0,1.3)$ & - \\
\hline & 1 & 20,021 & 269,637 & 38.2 & 0.031 & 0.766 & $1.5(1.4,1.7)$ & Ref \\
\hline & 2 & 42,287 & 902,497 & 1.6 & 0.424 & 0.325 & $1.6(1.5,1.6)$ & 0.784 \\
\hline Pregnancy & 3 & 8860 & 18,263 & 74.2 & 0.009 & 0.497 & $1.3(0.9,1.9)$ & 0.535 \\
\hline related & 5 & 771,027 & $14,009,374$ & 98.7 & 0.001 & 0.207 & $2.0(1.8,2.4)$ & 0.38 \\
\hline \multirow[t]{4}{*}{ Hypertension } & 6 & 42,762 & 959,991 & 76.4 & 0.005 & 0.051 & $2.1(1.7,2.6)$ & 0.160 \\
\hline & 7 & 751 & 18,674 & 0 & 0.471 & 0.484 & $1.8(1.3,2.5)$ & 0.248 \\
\hline & Overall & 886,089 & $1,618,008$ & 96.3 & 0.001 & 0.541 & $1.7(1.6,1.9)$ & - \\
\hline & 1 & 18,518 & 142,679 & 99.5 & 0.001 & 0.337 & $\begin{array}{c}-0.307 \\
(-0.560,-0.054)\end{array}$ & Ref \\
\hline \multirow{3}{*}{$\begin{array}{l}\text { Gestational } \\
\text { weight gain }\end{array}$} & 5 & 14,689 & 257,901 & 90 & 0.001 & 0.624 & $\begin{array}{c}-0.353 \\
(-0.569,-0.137)\end{array}$ & 0.911 \\
\hline & 7 & 2410 & 45,271 & 84.7 & 0.001 & 1.000 & $\begin{array}{c}-0.400 \\
(-0.567,-0.233)\end{array}$ & 0.988 \\
\hline & Overall & 35,714 & 447,958 & 99.4 & 0.001 & 0.564 & $\begin{array}{c}-0.333 \\
(-0.492,-0.174)\end{array}$ & - \\
\hline
\end{tabular}

* Effect size represents the odds ratio for all variables, except for weight gain that is the standardized mean difference. ${ }^{£}$ Analysis was not performed in all subgroups of GDM classifications due to insufficient data. ${ }^{€}$ As there were not enough studies in the first classification, the second one as a reference group for comparison was used.

The odds ratio of primary cesarean among women with GDM, regardless of GDM classification, was 1.4 folds greater than in healthy controls (Pooled overall OR $=1.4,95 \%$ CI: 1.2, 1.5) (Figure 2). 


\section{Primary CS}

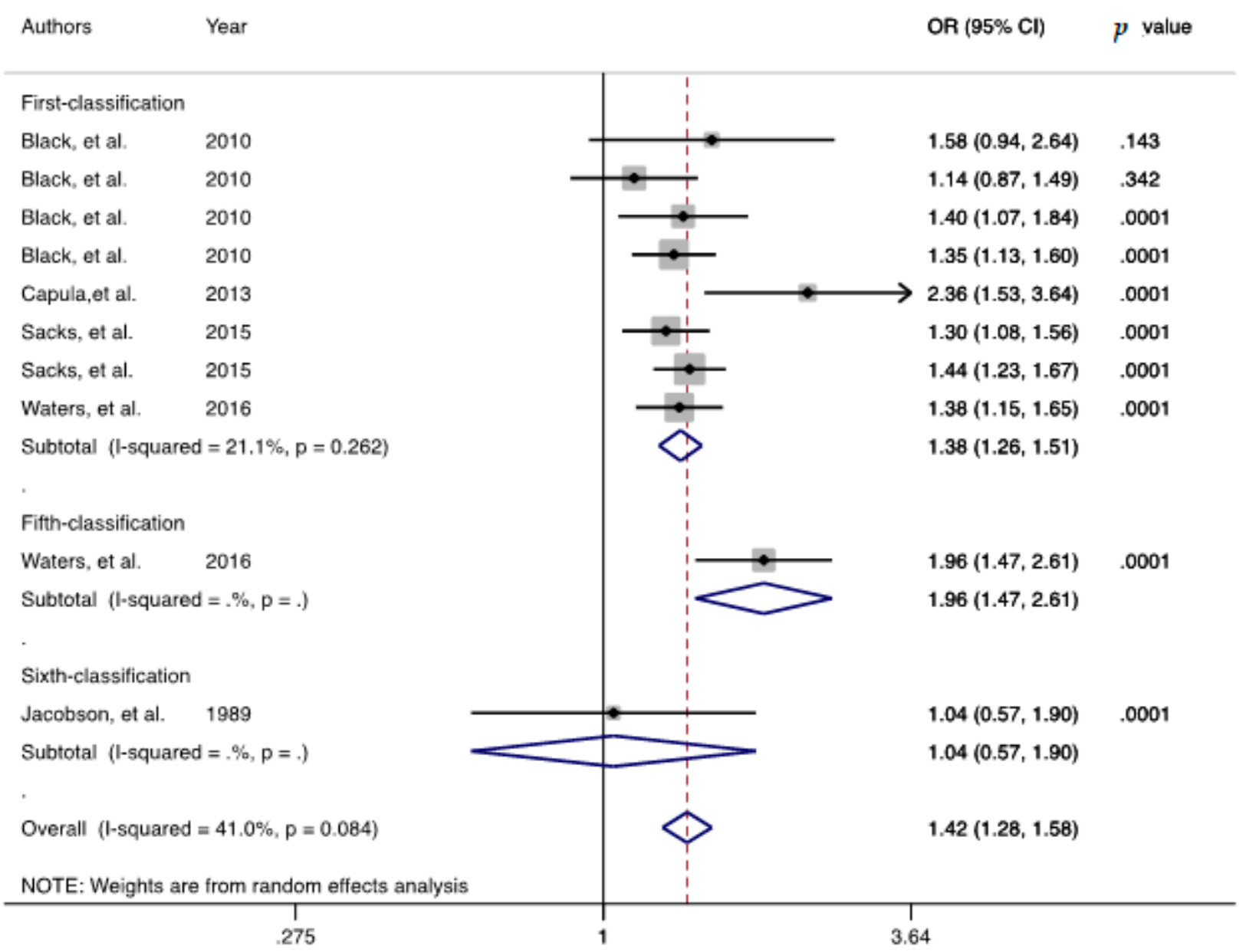

Figure 2. Meta-analysis forest plot of odds ratio (OR) OR for primary cesarean in women with and without Gestational Diabetes Mellitus (GDM) based on different diagnostic criteria.

In addition, risk of other adverse maternal outcomes, including induction of labor (Pooled overall OR = 1.7, 95\% CI: 1.6, 1.9), maternal hemorrhage (Pooled overall OR = 1.2, 95\% CI: 1.0, 1.3), and pregnancy-related hypertension (Pooled overall OR = 1.7, 95\% CI: 1.6, 1.9) among women with GDM, regardless of GDM diagnostic classification, were significantly higher than non-GDM counterparts (Table 2, Figures 3-5). 


\section{Induction of labor}

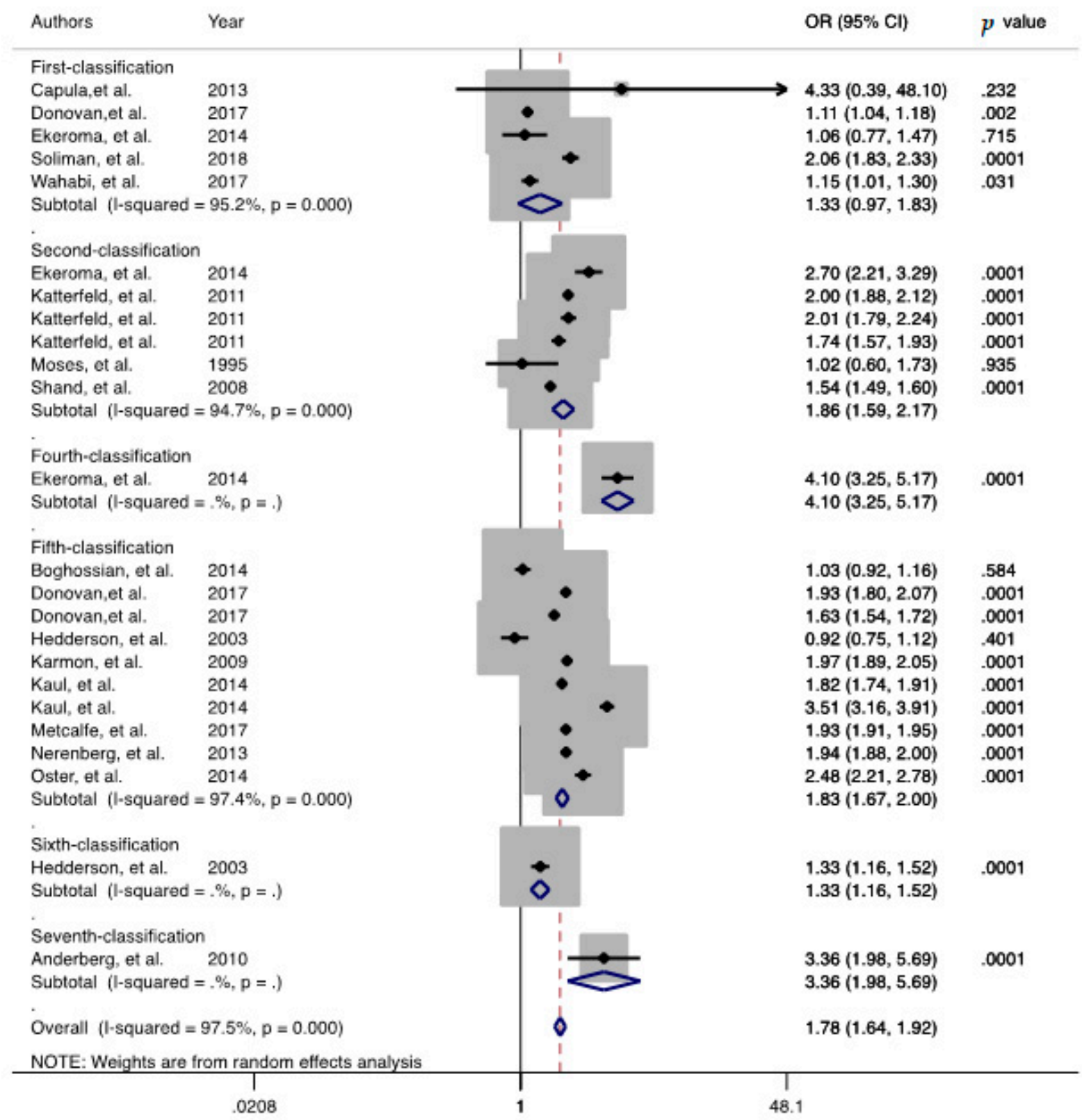

Figure 3. Meta-analysis forest plot of OR for the induction of labor among women with and without GDM based on different diagnostic criteria. 


\section{Maternal hemorrhage}

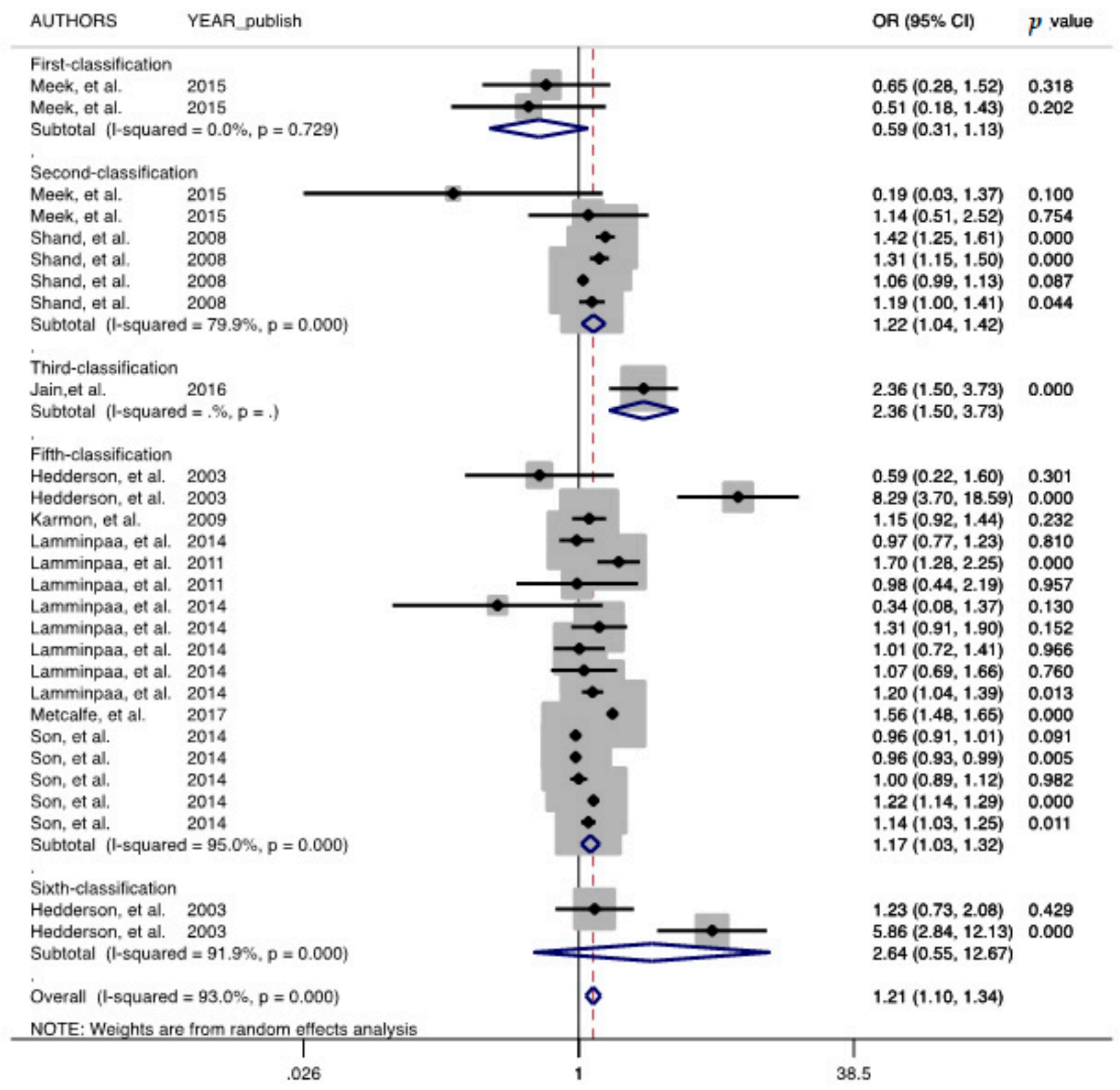

Figure 4. Meta-analysis forest plot of OR for maternal hemorrhage among women with and without GDM based on different diagnostic criteria. 


\section{Hypertension}

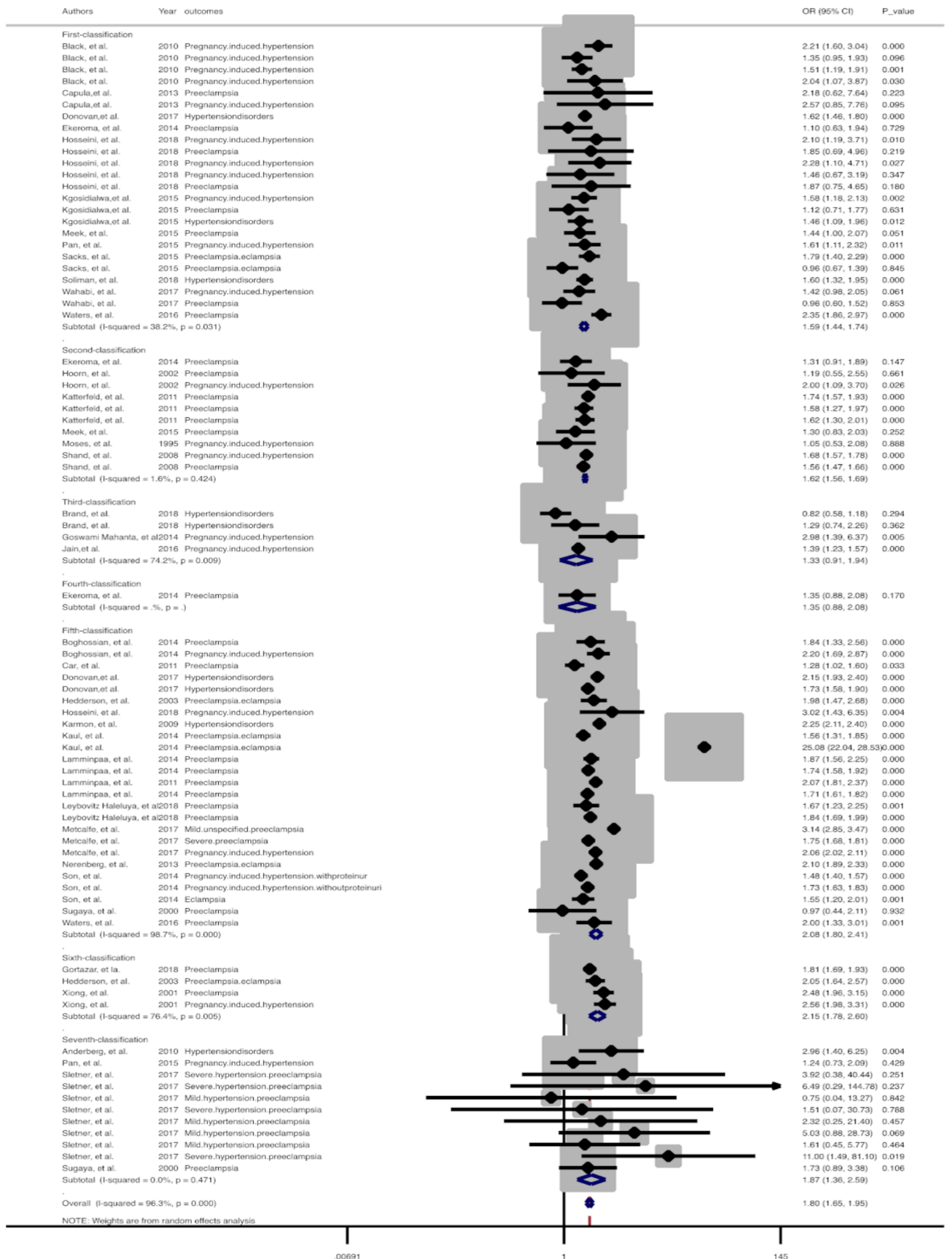

Figure 5. Meta-analysis forest plot of OR for pregnancy-related hypertension among women with and without GDM based on different diagnostic criteria. 
The gestational weight gain among women with GDM was significantly lower than the non-GDM population, (Pooled overall mean difference $=-0.333,95 \%$ CI $(-0.492$, -0.174) (Figure 6).

\section{Gestational Weight Gain}

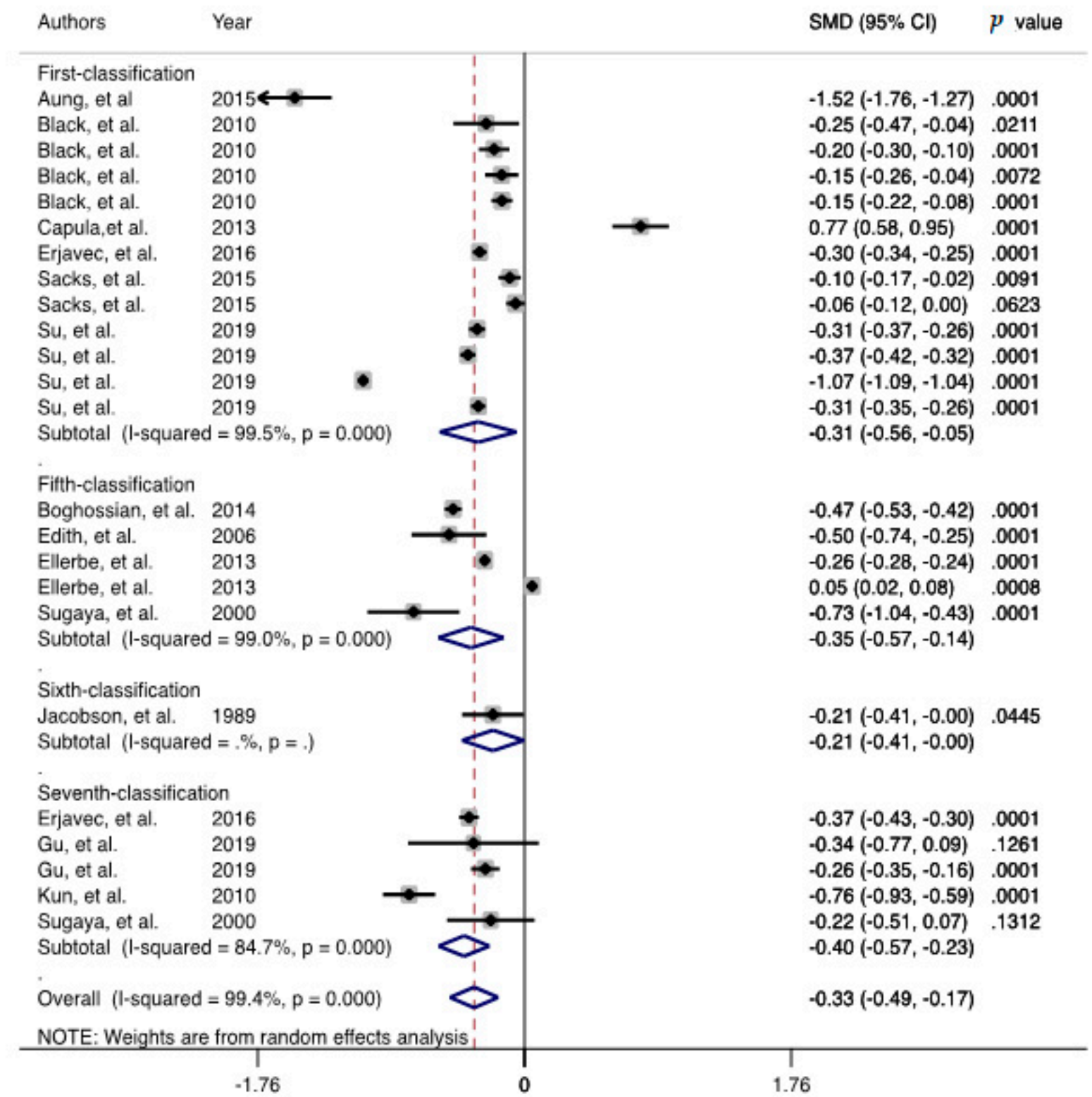

Figure 6. Meta-analysis forest plot of the mean difference of gestational weight gain among women with and without GDM based on different diagnostic criteria.

Subgroup analysis revealed that the risk of adverse maternal outcomes in women with GDM in all GDM diagnostic classifications were significantly higher than the non-GDM population (Table 2).

The results of meta-regression showed that the odds ratio/mean difference were notinfluenced by GDM diagnostic classification. The risk of adverse maternal outcomes in the IADPSG criteria classification, as the strictest criteria, was similar to others (Figure 7). 

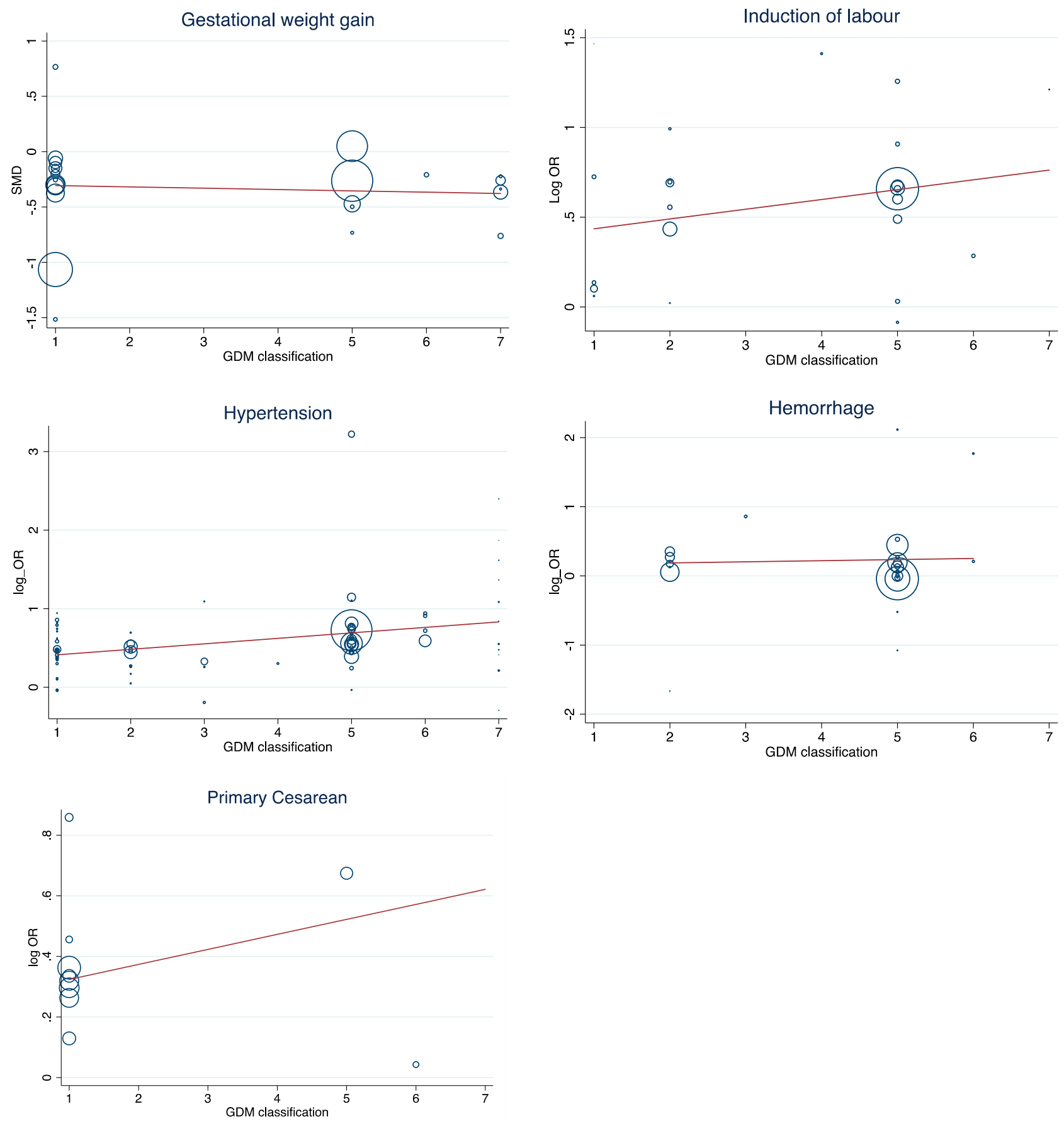

Figure 7. Bubble plot of the meta-regression relationships adverse outcomes and GDM classification.

\subsection{Results of Publication Bias and Risk of Bias evaluation}

According to Begg's test, no considerable publication bias for various meta-analyses was observed (Table 2). Results of the Risk of Bias evaluation are presented in Supplementary Figures S1A,B and S2A,B. Given that all included studies were observational, the overall risk of bias was low or probably low. However, half of the cross-sectional studies had a probably high risk of bias in the control of prognostic variables. $10 \%$ of cohort studies had a probable or high risk of bias in the assessment of exposure and bias in controlling prognostic variables. 


\section{Discussion}

Results of this systematic review and meta-analysis demonstrated that GDM, regardless of its diagnostic classification, could increase the risk of adverse maternal outcomes; however, the key finding is that, despite variations in screening approaches, screening methods, and diagnostic threshold values, the increased risk was not influenced by the GDM diagnostic classification.

Despite the wide range of endorsements and guidelines for the diagnosis of GDM in pregnant women recommended by international societies [1,13,69-74], there is a strong controversy over the definition of GDM including advice on selective approaches such as universal or risk-based screening, the optimal time for screening in the first and second trimesters, appropriate screening method or criteria for diagnosis, and proper threshold values. Furthermore, there are ongoing debates concerning the risk of adverse pregnancy outcomes and the cost-effectiveness of different screening or diagnostic strategies. However, the aim of almost six decades of research and tremendous efforts has been to reach a global consensus and uniformly accepted guideline with regard to the optimum and cost-effective approach for screening by which the risk of adverse pregnancy outcome is reduced.

The risk of adverse perinatal events using two main GDM diagnostic criteria has been studied by previous reviews. Given that our systematic review and meta-analysis compared all available criteria, it can have a complementary role to the findings of other reviews. For instance, Wendland et al. (2012) [6] in a systematically review and metaanalysis of the relationship between GDM based on the WHO and IADPSG criteria, and adverse events of preeclampsia and cesarean delivery, reported that these criteria could identify women with an elevated risk of adverse perinatal events. The same magnitude for both criteria was reported in our review. Another meta-analysis by Hosseini et al. (2018) [15] assessed the magnitude of the association between GDM using the IADPSG or Carpenter and Coustan criteria and selected adverse perinatal events. They demonstrated that the risk of adverse pregnancy events including preeclampsia, cesarean section, and gestational hypertension increased in both GDM criteria. Although associations with the Carpenter and Coustan criteria were slightly greater, it was not confirmed by the statistical test.

The results of our review demonstrated that despite an increased risk of adverse maternal outcomes among women with GDM, this risk had a similar magnitude for all GDM diagnostic classification. Considering that the use of the strict IADPSG criteria has a significant impact on health care costs and infrastructure capacity with a similar magnitude on short term adverse maternal outcomes, the cost-effectiveness of their use should be defined. Until now, there are not sufficient data to demonstrate the cost-effectiveness superiority of one screening and diagnostic approach over the other $[75,76]$. In addition, most available cost-effectiveness studies [75,77-80] were performed in developed societies with higher health economic resources and a lower rate of annual birth than developing and transitional countries [81].

Moreover, the label of GDM, its exhausting treatment, concerns about pregnant women, and unborn health status are some sources of stress, which may lead to a serious psychological problem for some pregnant women and families and could diminish the quality of life [82-84]. However, using the optimum cost-effective GDM diagnosis approach with an improved adverse outcome such problems can be prevented.

It is believed that GDM is associated with adverse perinatal events and our metaanalysis confirmed the findings of available literature. Diagnosis of GDM is associated with more pregnancy-related hypertension, and higher rates of induction of labor and primary cesarean section, irrespective of the diagnostic criteria used for GDM. However, insulin resistance has also been hypothesized to contribute to the pathophysiology of adverse outcomes [85]. In our review despite the lower gestational weight gain, an increase in the rate of primary cesarean was seen, which was associated with GDM and an increase in the frequency of induction of labor. It is assumed that gestational weight gain may not be the important factor responsible for the higher odds of cesarean section or induction 
of labor among women with GDM compared to non-GDM counterparts [7]. Fetal size and macrosomia given fetal insulin response to the elevated glucose level in the body of pregnant women or overtreatment may be associated with an elevated prevalence of cesarean section $[7,86]$. Moreover, the label of GDM can lead to a tendency toward cesarean section.

Ass the limitations of this review, studies that used the universal screening strategy were selected for inclusion in the meta-analysis. Therefore, studies from north Europe with a low prevalence of GDM that might use a targeted high-risk screening strategy were not included in our review. The short-term maternal outcomes of GDM were considered in our review indicating the need to evaluate the long-term adverse outcomes of GDM based on different diagnostic criteria. Also, given the lack of data on some GDM diagnostic criteria, subgroup analysis for classifications could not be carried out and the lack of a unique definition for each adverse pregnancy outcome may have affected our review findings and their generalizability. Additionally, the effect of diagnostic criteria on outcomes irrespective of GDM treatment strategies might have influenced the results.

\section{Conclusions}

The use of the straighten criteria of the IAPDSG definition can increase the prevalence of GDM among pregnant women. Also, the magnitude of the increased risk of adverse maternal outcomes in all diagnostic criteria was similar. The finding of our review can empower health care providers to select the cost-effective GDM screening approach for pregnant women.

Supplementary Materials: The following are available online at https:/ /www.mdpi.com/2077-0 383/10/4/666/s1, Table S1: Quality assessment of studies using the Newcastle-Ottawa Quality Assessment Scale for cohort studies., Table S2: Quality assessment of included studies using the Newcastle-Ottawa Quality Assessment Scale for cross-sectional study, Figure S1: Risk of bias in cross-sectional studies, Figure S2: Risk of bias in cohort studies.

Author Contributions: Conceptualization, F.R.T. and S.B.-G.; methodology, F.R.T. and S.B.-G.; software, M.S.G.N. and R.B.Y.; formal analysis, R.B.Y. and F.R.T.; investigation, M.S.G.N., S.B.-G. and F.R.T.; data curation, F.R.T., S.B.-G. and M.S.G.N.; writing-original draft preparation, F.R.T. and M.S.G.N.; writing—-review and editing, S.B.-G. and R.B.Y.; supervision, F.R.T. and S.B.-G.; project administration, F.R.T.; funding acquisition, S.B.-G. All authors have read and agreed to the published version of the manuscript.

Funding: This research was funded by National Institutes for Medical Research Development (NIMAD), grant number 972438 .

Institutional Review Board Statement: Not applicable.

Informed Consent Statement: Not applicable.

Data Availability Statement: The data presented in the study are available on request from the corresponding author.

Acknowledgments: The authors would like to thank Marzieh Atashkar, the library staff of the Research Institute for Endocrine Sciences, for assistance with the literature search. Also, Nord University, Bodø, Norway covered the article processing charges.

Conflicts of Interest: The authors declare no conflict of interest.

\section{References}

1. American Diabetes Association. Classification and Diagnosis of Diabetes: Standards of Medical Care in Diabetes-2020. Diabetes Care 2020, 43, S14-S31. [CrossRef]

2. Behboudi-Gandevani, S.; Amiri, M.; Yarandi, R.B.; Tehrani, F.R. The impact of diagnostic criteria for gestational diabetes on its prevalence: A systematic review and meta-analysis. Diabetol. Metab. Syndr. 2019, 11, 1-18. [CrossRef]

3. Gabbay-Benziv, R.; Doyle, L.E.; Blitzer, M.; Baschat, A.A. First trimester prediction of maternal glycemic status. J. Périnat. Med. 2015, 43, 283-289. [CrossRef] 
4. Giannakou, K.; Evangelou, E.; Yiallouros, P.; Christophi, C.A.; Middleton, N.; Papatheodorou, E.; Papatheodorou, S.I. Risk factors for gestational diabetes: An umbrella review of meta-analyses of observational studies. PLoS ONE 2019, 14, e0215372. [CrossRef]

5. Plows, J.F.; Stanley, J.L.; Baker, P.; Reynolds, C.M.; Vickers, M.H. The Pathophysiology of Gestational Diabetes Mellitus. Int. J. Mol. Sci. 2018, 19, 3342. [CrossRef]

6. Morikawa, M.; Sugiyama, T.; Sagawa, N.; Hiramatsu, Y.; Ishikawa, H.; Hamada, H.; Kameda, T.; Hara, E.; Toda, S.; Minakami, H. Perinatal mortality in Japanese women diagnosed with gestational diabetes mellitus and diabetes mellitus. J. Obstet. Gynaecol. Res. 2017, 43, 1700-1707. [CrossRef] [PubMed]

7. Gorgal, R.; Gonçalves, E.; Barros, M.; Namora, G.; Magalhães, Â.; Rodrigues, T.; Montenegro, N. Gestational diabetes mellitus: A risk factor for non-elective cesarean section. J. Obstet. Gynaecol. Res. 2011, 38, 154-159. [CrossRef]

8. Corrado, F.; D'Anna, R.; Laganà, A.S.; Di Benedetto, A. Abnormal glucose tolerance later in life in women affected by glucose intolerance during pregnancy. J. Obstet. Gynaecol. 2014, 34, 123-126. [CrossRef] [PubMed]

9. Vitagliano, A.; Saccone, G.; Cosmi, E.; Visentin, S.; Dessole, F.; Ambrosini, G.; Berghella, V. Inositol for the prevention of gestational diabetes: A systematic review and meta-analysis of randomized controlled trials. Arch. Gynecol. Obstet. 2019, 299, 55-68. [CrossRef] [PubMed]

10. Facchinetti, F.; Appetecchia, M.; Aragona, C.; Bevilacqua, A.; Espinola, M.S.B.; Bizzarri, M.; D'Anna, R.; Dewailly, D.; DiamantiKandarakis, E.; Marín, I.H.; et al. Experts' opinion on inositols in treating polycystic ovary syndrome and non-insulin dependent diabetes mellitus: A further help for human reproduction and beyond. Expert Opin. Drug Metab. Toxicol. 2020, 16, 255-274. [CrossRef]

11. Jacklin, P.B.; Maresh, M.J.; Patterson, C.C.; Stanley, K.P.; Dornhorst, A.; Burman-Roy, S.; Bilous, R.W. A cost-effectiveness comparison of the NICE 2015 and WHO 2013 diagnostic criteria for women with gestational diabetes with and without risk factors. BMJ Open 2017, 7, e016621. [CrossRef] [PubMed]

12. Metzger, B.E.; Lowe, L.; Dyer, A.; Trimble, E.; Chaovarindr, U.; Coustan, D.; Hadden, D.; McCance, D.; Hod, M.; McIntyre, H.; et al. Hyperglycemia and Adverse Pregnancy Outcomes. Obstet. Anesth. Dig. 2009, 29, 39-40. [CrossRef]

13. International Association of Diabetes and Pregnancy Study Groups Consensus Panel. International association of diabetes and pregnancy study groups recommendations on the diagnosis and classification of hyperglycemia in pregnancy. Diabetes Care 2010, 33, 676-682. [CrossRef] [PubMed]

14. World Health Organization. Diagnostic Criteria and Classification of Hyperglycaemia First Detected in Pregnancy; World Health Organization: Geneva, Switzerland, 2013.

15. Hosseini, E.; Janghorbani, M. Systematic review and meta-analysis of diagnosing gestational diabetes mellitus with one-step or two-step approaches and associations with adverse pregnancy outcomes. Int. J. Gynaecol. Obstet. 2018, 143, 137-144. [CrossRef] [PubMed]

16. Moher, D.; Liberati, A.; Tetzlaff, J.; Wong, C.S. Preferred reporting items for systematic reviews and meta-analyses: The PRISMA statement. Int. J. Sur. 2010, 8, 336-341. [CrossRef]

17. Stang, A. Critical evaluation of the Newcastle-Ottawa scale for the assessment of the quality of nonrandomized studies in meta-analyses. Eur. J. Epidemiol. 2010, 25, 603-605. [CrossRef] [PubMed]

18. Sterne, J.A.; Hernán, M.A.; Reeves, B.C.; Savović, J.; Berkman, N.D.; Viswanathan, M.; Henry, D.; Altman, D.G.; Ansari, M.T.; Boutron, I.; et al. ROBINS-I: A tool for assessing risk of bias in non-randomised studies of interventions. BMJ 2016, 355 , i4919. [CrossRef]

19. Higgins, J. Analysing data and undertaking meta-analyses In Cochrane Handbook for Systematic Reviews of Interventions (Version 5.1. 0); Higgins, J., Green, S., Eds.; Wiley: New York, NY, USA, 2011.

20. Shand, A.W.; Bell, J.C.; McElduff, A.; Morris, J.; Roberts, C.L. Outcomes of pregnancies in women with pre-gestational diabetes mellitus and gestational diabetes mellitus; a population-based study in New South Wales, Australia, 19982002. Diabet. Med. 2008, 25, 708-715. [CrossRef] [PubMed]

21. Erjavec, K.; Poljičanin, T.; Matijević, R. Impact of the Implementation of New WHO Diagnostic Criteria for Gestational Diabetes Mellitus on Prevalence and Perinatal Outcomes: A Population-Based Study. J. Pregnancy 2016, 2016, 1-6. [CrossRef] [PubMed]

22. Black, M.H.; Sacks, D.A.; Xiang, A.H.; Lawrence, J.M. Clinical Outcomes of Pregnancies Complicated by Mild Gestational Diabetes Mellitus Differ by Combinations of Abnormal Oral Glucose Tolerance Test Values. Diabetes Care 2010, 33, 2524-2530. [CrossRef]

23. Lamminpää, R.; Vehviläinen-Julkunen, K.; Gissler, M.; Gissler, M.; Selander, T.; Heinonen, S. Pregnancy outcomes in women aged 35 years or older with gestational diabetes-A registry-based study in Finland. J. Matern. Fetal Neonatal Med. 2016, 29, 55-59. [CrossRef] [PubMed]

24. Carr, D.B.; Newton, K.M.; Utzschneider, K.M.; Faulenbach, M.V.; Kahn, S.E.; Easterling, T.R.; Heckbert, S.R. Gestational Diabetes or Lesser Degrees of Glucose Intolerance and Risk of Preeclampsia. Hypertens. Pregnancy 2010, 30, 153-163. [CrossRef]

25. Metcalfe, A.; Sabr, Y.; Hutcheon, J.A.; Donovan, L.; Lyons, J.; Burrows, J.; Joseph, K.S. Trends in Obstetric Intervention and Pregnancy Outcomes of Canadian Women with Diabetes in Pregnancy From 2004 to 2015. J. Endocr. Soc. 2017, 1, 1540-1549. [CrossRef] [PubMed]

26. Su, W.-J.; Chen, Y.-L.; Huang, P.-Y.; Shi, X.-L.; Yan, F.-F.; Chen, Z.; Yan, B.; Song, H.-Q.; Lin, M.-Z.; Li, X. Effects of Prepregnancy Body Mass Index, Weight Gain, and Gestational Diabetes Mellitus on Pregnancy Outcomes: A Population-Based Study in Xiamen, China, 2011-2018. Ann. Nutr. Metab. 2019, 75, 31-38. [CrossRef] [PubMed] 
27. Van Hoorn, J.; Dekker, G.; Jeffries, B. Gestational diabetes versus obesity as risk factors for pregnancy-induced hypertensive disorders and fetal macrosomia. Aust. N. Z. J. Obstet. Gynaecol. 2002, 42, 35-40. [CrossRef] [PubMed]

28. Zeki, R.; Oats, J.J.; Wang, A.Y.; Li, Z.; Homer, C.S.E.; Sullivan, E.A. Cesarean section and diabetes during pregnancy: An NSW population study using the Robson classification. J. Obstet. Gynaecol. Res. 2018, 44, 890-898. [CrossRef]

29. Sugaya, A.; Sugiyama, T.; Nagata, M.; Toyoda, N. Comparison of the validity of the criteria for gestational diabetes mellitus by WHO and by the Japan Society of Obstetrics and Gynecology by the outcomes of pregnancy. Diabetes Res. Clin. Pr. 2000, 50, 57-63. [CrossRef]

30. Sletner, L.; Jenum, A.K.; Yajnik, C.S.; Mørkrid, K.; Nakstad, B.; Rognerud-Jensen, O.H.; Birkeland, K.I.; Vangen, S. Fetal growth trajectories in pregnancies of European and South Asian mothers with and without gestational diabetes, a population-based cohort study. PLOS ONE 2017, 12, e0172946. [CrossRef]

31. Ellerbe, C.N.; Gebregziabher, M.; Korte, J.E.; Mauldin, J.; Hunt, K.J. Quantifying the Impact of Gestational Diabetes Mellitus, Maternal Weight and Race on Birthweight via Quantile Regression. PLoS ONE 2013, 8, e65017. [CrossRef]

32. Mahanta, T.G.; Deuri, A.; Mahanta, B.N.; Bordoloi, P.; Rasaily, R.; Mahanta, J.; Baruah, S.; Gogoi, P. Maternal and foetal outcome of gestational diabetes mellitus in a rural block of Assam, India. Clin. Epidemiol. Glob. Health 2014, 2, 9-15. [CrossRef]

33. Kieffer, E.C.; Tabaei, B.P.; Carman, W.J.; Nolan, G.H.; Guzman, J.R.; Herman, W.H. The Influence of Maternal Weight and Glucose Tolerance on Infant Birthweight in Latino Mother-Infant Pairs. Am. J. Public Health 2006, 96, 2201-2208. [CrossRef] [PubMed]

34. Nerenberg, K.A.; Johnson, J.A.; Leung, B.; Savu, A.; Ryan, E.A.; Chik, C.L.; Kaul, P. Risks of Gestational Diabetes and Preeclampsia Over the Last Decade in a Cohort of Alberta Women. J. Obstet. Gynaecol. Can. 2013, 35, 986-994. [CrossRef]

35. Soliman, A.; Salama, H.; Al Rifai, H.; De Sanctis, V.; Al-Obaidly, S.; Al Qubasi, M.; Olukade, T. The effect of different forms of dysglycemia during pregnancy on maternal and fetal outcomes in treated women and comparison with large cohort studies. Acta Biomed. Atenei Parm. 2018, 89, 11-21.

36. Oster, R.T.; King, M.; Morrish, D.W.; Mayan, M.; Toth, E.L. Diabetes in pregnancy among First Nations women in Alberta, Canada: A retrospective analysis. BMC Pregnancy Childbirth 2014, 14, 136. [CrossRef]

37. Xiong, X.; Saunders, L.; Wang, F.; Demianczuk, N. Gestational diabetes mellitus: Prevalence, risk factors, maternal and infant outcomes. Int. J. Gynecol. Obstet. 2001, 75, 221-228. [CrossRef]

38. Sacks, D.A.; Black, M.H.; Li, X.; Montoro, M.N.; Lawrence, J.M. Adverse pregnancy outcomes using the International Association of the Diabetes and Pregnancy Study Groups criteria: Glycemic thresholds and associated risks. Obstet. Gynecol. 2015, 126, 67-73. [CrossRef]

39. Son, K.H.; Lim, N.K.; Lee, J.; Cho, M.; Park, H. Comparison of maternal morbidity and medical costs during pregnancy and delivery between patients with gestational diabetes and patients with pre-existing diabetes. Diabet. Med. 2015, 32, 477-486. [CrossRef]

40. Pan, L.; Leng, J.; Liu, G.; Zhang, C.; Liu, H.; Li, M.; Tan, L.; Tian, H.; Chan, J.C.; Hu, G.; et al. Pregnancy outcomes of Chinese women with gestational diabetes mellitus defined by the IADPSG's but not by the 1999 WHO's criteria. Clin. Endocrinol. 2015, 83, 684-693. [CrossRef]

41. Jacobson, J.D.; Cousins, L. A population-based study of maternal and perinatal outcome in patients with gestational diabetes. Am. J. Obstet. Gynecol. 1989, 161, 981-986. [CrossRef]

42. Leybovitz-Haleluya, N.; Wainstock, T.; Landau, D.; Sheiner, E. Maternal gestational diabetes mellitus and the risk of subsequent pediatric cardiovascular diseases of the offspring: A population-based cohort study with up to 18 years of follow up. Acta Diabetol. 2018, 55, 1037-1042. [CrossRef] [PubMed]

43. Gortazar, L.; Roux, J.A.F.-L.; Benaiges, D.; Sarsanedas, E.; Payà, A.; Mañé, L.; Pedro-Botet, J.; Goday, A. Trends in prevalence of gestational diabetes and perinatal outcomes in Catalonia, Spain, 2006 to 2015: The Diagestcat Study. Diabetes Metabol. Res. Rev. 2019, 35, e3151. [CrossRef] [PubMed]

44. Hedderson, M.M.; Ferrara, A.; Sacks, D.A. Gestational diabetes mellitus and lesser degrees of pregnancy hyperglycemia: Association with increased risk of spontaneous preterm birth. Obstet. Gynecol. 2003, 102, 850-856. [CrossRef]

45. Kun, A. Insulin Resistance Is Associated with Gestational Hypertension and Not with Preeclampsia: A Population-Based Screening Study. Gynecol. Obstet. Investig. 2011, 71, 256-261. [CrossRef]

46. Davey, S.; Jain, R.; Davey, A.; Raghav, S.K.; Singh, J.V. Can the management of blood sugar levels in gestational diabetes mellitus cases be an indicator of maternal and fetal outcomes? The results of a prospective cohort study from India. J. Fam. Community Med. 2016, 23, 94-99. [CrossRef]

47. Hosseini, E.; Janghorbani, M.; Aminorroaya, A. Incidence, risk factors, and pregnancy outcomes of gestational diabetes mellitus using one-step versus two-step diagnostic approaches: A population-based cohort study in Isfahan, Iran. Diabetes Res. Clin. Pr. 2018, 140, 288-294. [CrossRef] [PubMed]

48. Hosseini, E.; Janghorbani, M.; Shahshahan, Z. Comparison of risk factors and pregnancy outcomes of gestational diabetes mellitus diagnosed during early and late pregnancy. Midwifery 2018, 66, 64-69. [CrossRef]

49. Zamstein, O.; Sheiner, E.; Wainstock, T.; Landau, D.; Walfisch, A. Maternal gestational diabetes and long-term respiratory related hospitalizations of the offspring. Diabetes Res. Clin. Pr. 2018, 140, 200-207. [CrossRef]

50. Aung, Y.Y.M.; Sowter, M.; Kenealy, T.; Herman, J.; Ekeroma, A. Gestational diabetes mellitus screening, management and outcomes in the Cook Islands. N. Z. Med. J. 2015, 128. 
51. Ekeroma, A.J.; Chandran, G.S.; McCowan, L.; Ansell, D.; Eagleton, C.; Kenealy, T. Impact of using the international association of diabetes and pregnancy study groups criteria in South Auckland: Prevalence, interventions and outcomes. Aust. N. Z. J. Obstet. Gynaecol. 2014, 55, 34-41. [CrossRef]

52. Kieffer, E.C.; Nolan, G.H.; Carman, W.J.; Sanborn, C.Z.; Guzman, R.; Ventura, A. Glucose Tolerance During Pregnancy and Birth Weight in a Hispanic Population. Obstet. Gynecol. 1999, 94, 741-746. [CrossRef] [PubMed]

53. Donovan, L.; Edwards, A.; Savu, A.; Butalia, S.; Ryan, E.A.; Johnson, J.A.; Kaul, P. Population-level outcomes with a 2-step approach for gestational diabetes screening and diagnosis. Can. J. Diabetes 2017, 41, 596-602. [CrossRef]

54. Kgosidialwa, O.; Egan, A.M.; Carmody, L.; Kirwan, B.; Gunning, P.; Dunne, F.P. Treatment with Diet and Exercise for Women With Gestational Diabetes Mellitus Diagnosed Using IADPSG Criteria. J. Clin. Endocrinol. Metab. 2015, 100, 4629-4636. [CrossRef] [PubMed]

55. Kaul, P.; Savu, A.; Nerenberg, K.A.; Donovan, L.; Donovan, E.; Chik, C.L.; Ryan, E.A.; Johnson, J.A. Impact of gestational diabetes mellitus and high maternal weight on the development of diabetes, hypertension and cardiovascular disease: A population-level analysis. Diabet. Med. 2015, 32, 164-173. [CrossRef]

56. Brand, J.S.; West, J.; Tuffnell, D.; Bird, P.K.; Wright, J.; Tilling, K.; Lawlor, D.A. Gestational diabetes and ultrasound-assessed fetal growth in South Asian and White European women: Findings from a prospective pregnancy cohort. BMC Med. 2018, 16, 203. [CrossRef]

57. Kawakita, T.; Bowers, K.; Hazrati, S.; Zhang, C.; Grewal, J.; Chen, Z.; Sun, L.; Grantz, K.L.; Gtantz, K. Increased Neonatal Respiratory Morbidity Associated with Gestational and Pregestational Diabetes: A Retrospective Study. Am. J. Perinatol. 2017, 34, 1160-1168. [CrossRef] [PubMed]

58. Boghossian, N.S.; Yeung, E.; Albert, P.S.; Mendola, P.; Laughon, S.K.; Hinkle, S.N.; Zhang, C. Changes in diabetes status between pregnancies and impact on subsequent newborn outcomes. Am. J. Obstet. Gynecol. 2014, 210, 431.e1. [CrossRef] [PubMed]

59. Meek, C.L.; Lewis, H.B.; Patient, C.; Davies, G.A.; Poitras, V.; Gray, C.; Jaramillo Garcia, A.; Barrowman, N.; Adamo, K.B.; Duggan, M.; et al. Diagnosis of gestational diabetes mellitus: Falling through the net. Diabetologia 2015, 58, 2003-2012. [CrossRef] [PubMed]

60. Wahabi, H.; Fayed, A.; Esmaeil, S.; Mamdouh, H.; Kotb, R. Prevalence and complications of pregestational and gestational diabetes in Saudi women: Analysis from Riyadh Mother and Baby cohort study (RAHMA). Biomed. Res. Int. 2017, 2017, 6878263. [CrossRef]

61. Avalos, G.E.; Owens, L.A.; Dunne, F.; ATLANTIC DIP Collaborators. Applying current screening tools for gestational diabetes mellitus to a European population: Is it time for change? Diabetes Care 2013, 36, 3040-3044. [CrossRef]

62. Anderberg, E.; Källén, K.; Berntorp, K. The impact of gestational diabetes mellitus on pregnancy outcome comparing different cut-off criteria for abnormal glucose tolerance. Acta Obstet. Gynecol. Scand. 2010, 89, 1532-1537. [CrossRef]

63. Gu, Y.; Lu, J.; Li, W.; Liu, H.; Wang, L.; Leng, J.; Li, W.; Zhang, S.; Wang, S.; Tuomilehto, J.; et al. Joint Associations of Maternal Gestational Diabetes and Hypertensive Disorders of Pregnancy with Overweight in Offspring. Front. Endocrinol. 2019, 10. [CrossRef]

64. Waters, T.P.; Dyer, A.R.; Scholtens, D.M.; Dooley, S.L.; Herer, E.; Lowe, L.P.; Oats, J.J.; Persson, B.; Sacks, D.A.; HAPO Cooperative Study Research Group; et al. Maternal and Neonatal Morbidity for Women Who Would Be Added to the Diagnosis of GDM Using IADPSG Criteria: A Secondary Analysis of the Hyperglycemia and Adverse Pregnancy Outcome Study. Diabetes Care 2016, 39, 2204-2210. [CrossRef] [PubMed]

65. Moses, R.; Griffiths, R. Can a diagnosis of gestational diabetes be an advantage to the outcome of pregnancy? J. Soc. Gynecol. Investig. 1995, 2, 523-525. [CrossRef]

66. Karmon, A.; Levy, A.; Holcberg, G.; Wiznitzer, A.; Mazor, M.; Sheiner, E. Decreased perinatal mortality among women with diet-controlled gestational diabetes mellitus. Int. J. Gynaecol. Obstet. 2009, 104, 199-202. [CrossRef]

67. Capula, C.; Chiefari, E.; Vero, A.; Arcidiacono, B.; Iiritano, S.; Puccio, L.; Pullano, V.; Foti, D.P.; Brunetti, A.; Vero, R. Gestational Diabetes Mellitus: Screening and Outcomes in Southern Italian Pregnant Women. ISRN Endocrinol. 2013, 2013, 1-8. [CrossRef]

68. Von Katterfeld, B.; Li, J.; McNamara, B.; Langridge, A.T. Maternal and neonatal outcomes associated with gestational diabetes in women from culturally and linguistically diverse backgrounds in Western Australia. Diabet. Med. 2012, 29, 372-377. [CrossRef] [PubMed]

69. López Stewart, G. Diagnostic Criteria and Classification of Hyperglycaemia First Detected in Pregnancy: A World Health Organization Guideline; World Health Organization: Geneva, Switzerland, 2014.

70. Bogdanet, D.; O'Shea, P.M.; Lyons, C.; Shafat, A.; Dunne, F. The Oral Glucose Tolerance Test-Is It Time for a Change?-A Literature Review with an Emphasis on Pregnancy. J. Clin. Med. 2020, 9, 3451. [CrossRef] [PubMed]

71. Ansarzadeh, S.; Salehi, L.; Mahmoodi, Z.; Mohammadbeigi, A. Factors affecting the quality of life in women with gestational diabetes mellitus: A path analysis model. Health Qual. Life Outcomes 2020, 18, 1-9. [CrossRef]

72. Hoffman, L.; Nolan, C.; Wilson, J.D.; Oats, J.J.N.; Simmons, D. Gestational diabetes mellitus-Management guidelines: The Australasian Diabetes in Pregnancy Society. Med. J. Aust. 1998, 169, 93-97. [CrossRef] [PubMed]

73. ACOG. Gestational Diabetes Mellitus; ACOG Practice Bulletin; ACOG: Washington, DC, USA, 2018.

74. Lash, R.W. Diabetes and Pregnancy-An Endocrine Society Clinical Practice Guideline Publication Note. J. Clin. Endocrinol. Metab. 2018, 103, 4042. [CrossRef] [PubMed] 
75. Mission, J.F.; Ohno, M.S.; Cheng, Y.W.; Caughey, A.B. Gestational diabetes screening with the new IADPSG guidelines: A cost-effectiveness analysis. Am. J. Obstet. Gynecol. 2012, 207, 326.e1. [CrossRef]

76. Weile, L.K.; Kahn, J.G.; Marseille, E.; Jensen, D.M.; Damm, P.; Lohse, N. Global cost-effectiveness of GDM screening and management: Current knowledge and future needs. Best Pract. Res. Clin. Obstet. Gynaecol. 2015, 29, 206-224. [CrossRef]

77. Fitria, N.; Van Asselt, A.D.I.; Postma, M.J. Cost-effectiveness of controlling gestational diabetes mellitus: A systematic review. Eur. J. Health Econ. 2019, 20, 407-417. [CrossRef] [PubMed]

78. Moss, J.R.; Crowther, C.A.; Hiller, J.E.; Willson, K.J.; Robinson, J.S.; Australian Carbohydrate Intolerance Study in Pregnant Women Group. Costs and consequences of treatment for mild gestational diabetes mellitus-evaluation from the ACHOIS randomised trial. BMC Pregnancy Childbirth 2007, 7, 27. [CrossRef] [PubMed]

79. Ohno, M.S.; Sparks, T.N.; Cheng, Y.W.; Caughey, A.B. Treating mild gestational diabetes mellitus: A cost-effectiveness analysis. Am. J. Obstet. Gynecol. 2011, 205, 282.e1. [CrossRef]

80. Poncet, B.; Touzet, S.; Rocher, L.; Berland, M.; Orgiazzi, J.; Colin, C. Cost-effectiveness analysis of gestational diabetes mellitus screening in France. Eur. J. Obstet. Gynecol. Reprod. Biol. 2002, 103, 122-129. [CrossRef]

81. Kalra, S.; Baruah, M.P.; Gupta, Y.; Kalra, B. Gestational diabetes: An onomastic opportunity. Lancet Diabetes Endocrinol. $2013,1,91$. [CrossRef]

82. Marchetti, D.; Carrozzino, D.; Fraticelli, F.; Fulcheri, M.; Vitacolonna, E. Quality of Life in Women with Gestational Diabetes Mellitus: A Systematic Review. J. Diabetes Res. 2017, 2017, 1-12. [CrossRef] [PubMed]

83. Kalra, B.; Gupta, Y.; Baruah, M.P. Renaming gestational diabetes mellitus: A psychosocial argument. Indian J. Endocrinol. Metab. 2013, 17, 593-595. [CrossRef]

84. Pantzartzis, K.A.; Manolopoulos, P.P.; Paschou, S.A.; Kazakos, K.; Kotsa, K.; Goulis, D.G. Gestational diabetes mellitus and quality of life during the third trimester of pregnancy. Qual. Life Res. 2019, 28, 1349-1354. [CrossRef]

85. Weissgerber, T.L.; Mudd, L.M. Preeclampsia and diabetes. Curr. Diabetes Rep. 2015, 15, 1-10. [CrossRef] [PubMed]

86. Naylor, C.D.; Sermer, M.; Chen, E.; Sykora, K. Cesarean Delivery in Relation to Birth Weight and Gestational Glucose Tolerance. JAMA 1996, 275, 1165-1170. [CrossRef] [PubMed] 